\title{
Model Predictive Control Schemes for Consensus in Multi-Agent Systems with Single- and Double-Integrator Dynamics
}

\author{
Giancarlo Ferrari-Trecate, Member, IEEE, Luca Galbusera, Marco Pietro Enrico Marciandi, Riccardo Scattolini
}

\begin{abstract}
In this paper we address the problem of driving a group of agents towards a consensus point when the agents have a discrete-time single- or double-integrator dynamics and the communication network is time-varying. We propose decentralized Model Predictive Control (MPC) schemes that take into account constraints on the agents' input and show that they guarantee consensus under mild assumptions. Since the global cost does not decrease monotonically, it cannot be used as a Lyapunov function for proving convergence to consensus. For this reason, our proofs exploit geometric properties of the optimal path followed by individual agents.
\end{abstract}

Index Terms-Networked autonomous agents, Consensus problems, Decentralized model predictive control.

\section{INTRODUCTION}

$\mathbf{T}$ HIS paper deals with consensus problems for dynamically decoupled agents described by a discrete-time single- or double-integrator model and subject to possible time-varying interconnections. The objective is to define decentralized control strategies guaranteeing that the states of all agents converge to a common value, called consensus point, which generally speaking depends on the agents' state and on the communication network. This problem is relevant in many fields, e.g. in computer graphics, unmanned autonomous vehicles, sensor networks and, generally speaking, in the control of cooperating systems (see [1] and the references therein; see also [2] for a more general definition of consensus in presence of unknown but bounded disturbances).

In recent years, many control laws have been proposed for networks of dynamic agents with different models and communication topologies, see e.g. [3], [4], [5], [6], [7], [8], [9]. Most of them do not exploit optimal control ideas and, with the exception of [3] and [7], do not account for input constraints, which in many cases have to be included in the problem formulation due to actuators limitations.

In this paper we propose innovative solutions based on Model Predictive Control (MPC), which is a widely used approach in view of its ability to handle control and state constraints. This method can be applied in a distributed fashion to the control of a group of agents by letting each agent solve, at each step, a constrained finite-time optimal control problem involving the state of neighboring agents. Moreover, following the so-called Receding-Horizon principle, at each time step the controller

G. Ferrari-Trecate is with the Dipartimento di Informatica e Sistemistica, Università degli Studi di Pavia, Via Ferrata 1, 27100 Pavia, Italy. e-mail: giancarlo.ferrari@unipv.it

L. Galbusera and R. Scattolini are with the Dipartimento di Elettronica e Informazione, Politecnico di Milano, Piazza Leonardo da Vinci 32, 20133 Milano, Italy. e-mail: \{galbusera, scattolini\}eelet.polimi.it

M.P.E. Marciandi is with CESI Ricerca S.p.a., Via Rubattino 54, 20134 Milano, Italy. e-mail: mmarciandi@hotmail.com

Corresponding author: G. Ferrari-Trecate. only applies the first input of the computed control sequence. Stabilizing MPC algorithms for decentralized and distributed systems have been proposed in [10], [11], [12], [13], [14], [15], [16] for dynamically coupled and decoupled systems. However these approaches heavily rely on the fact that all agents $a$ priori know a common set point, with the notable exception of [17], where the properties of the proposed algorithm are demonstrated by means of simulation experiments. On the contrary, the methods described in this paper can be formally proved to guarantee (asymptotic) consensus under control constraints and for time-varying communication networks.

Specifically, we first propose an MPC solution for consensus when agents have a single-integrator dynamics with bounded inputs. Notably, the proof of consensus under this scheme does not rely on the standard arguments used in predictive control to guarantee closed-loop stability, see e.g. [18], [19], since the global cost to be minimized by the MPC algorithm is not monotonically decreasing and, as such, it cannot be used as a Lyapunov function. Rather, we exploit geometric properties of the optimal path followed by individual agents and rely on the general results described in [3] for analyzing consensus. As a further matter, on the basis of this technique we develop other MPC solutions for consensus. We first modify the cost function adopted in the previous scheme and add a specific constraint on the state of the system. This constraint is called "contractive" because it mimics, in a multi-agent system domain, the state constraint proposed in [20] for the control of nonlinear systems. The main advantage of this approach is that it can be extended to agents with double-integrator dynamics, even though the techniques used for proving consensus in this case are more sophisticated.

In our schemes, because of the Receding-Horizon technique, the value of the consensus point depends not only on the initial conditions of the system, but also on the sequence of agents' states along time and on the communication network along time.

The paper is organized as follows: Section II is devoted to the definition of a model for the communication network and summarizes some key results on convergence in multi-agent systems presented in [3] and used in this paper. Section III describes the first MPC technique we propose for consensus in a network of integrators with time-varying communication. The contractive MPC solutions for single- and double-integrators are presented, respectively, in Sections IV and V. Simulation examples confirm the results obtained by applying the control laws we propose. Section VI is devoted to conclusions. Finally, the Appendix contains technical results used in the proofs of consensus. 


\section{BASIC NOTIONS AND PRELIMINARY RESULTS}

We consider a team of $n$ agents indexed by the elements of the set $\mathcal{N}_{G}=\{1, \ldots, n\}$. The communication network is represented by a weighted directed graph $G=\left(\mathcal{N}_{G}, \mathcal{E}_{G}, w_{G}\right)$, where $\mathcal{E}_{G} \subseteq\left\{(i, j): i, j \in \mathcal{N}_{G}, j \neq i\right\}$ is the set of edges and $w_{G}: \mathcal{E}_{G} \mapsto \mathbb{R}_{>0}$ associates to each edge $(i, j) \in \mathcal{E}_{G}$ a strictly positive weight denoted by $w_{i j}$. $G$ is bidirectional if $(i, j) \in \mathcal{E}_{G} \Leftrightarrow(j, i) \in \mathcal{E}_{G}{ }^{1}$ and unweighted if, $\forall(i, j) \in \mathcal{E}_{G}$, $w_{i j}=1$. In the latter case, the graph can be simply represented by the pair $\left(\mathcal{N}_{G}, \mathcal{E}_{G}\right)$. From now on we will generally refer to directed or bidirectional graphs assuming implicitly that they can be weighted. If, $\forall i, j \in \mathcal{N}_{G},(i, j) \in \mathcal{E}_{G}$, the graph is complete.

A node $i \in \mathcal{N}_{G}$ is connected to a node $j \in \mathcal{N}_{G} \backslash\{i\}$ if there is a path from $i$ to $j$ in the graph following the orientation of the arcs. The graph $G$ is strongly connected if, $\forall(i, j) \in$ $\mathcal{N}_{G} \times \mathcal{N}_{G}, i$ is connected to $j$. A directed graph is said to have a spanning tree if and only if $\exists i \in \mathcal{N}_{G}$ such that there is a path from $i$ to any other node $j \in \mathcal{N}_{G}$.

The creation and loss of communication links can be modeled by means of a time-dependent collection of graphs $\mathcal{G}=\left\{G(k)=\left(\mathcal{N}_{G}, \mathcal{E}_{G}(k), w_{G}(k)\right), k \in \mathbb{N}\right\}$ where all graphs $G(k)$ share the same set of nodes. $\mathcal{G}$ is a collection of bidirectional graphs if and only if, $\forall k \in \mathbb{N}, G(k)$ is bidirectional. Otherwise, it is referred to as a collection of directed graphs.

In the sequel, discrete-time intervals will be denoted with $\left[k_{1}, k_{2}\right]=\left\{k: k_{1} \leq k \leq k_{2}\right\}$ assuming implicitly that $0 \leq$ $k_{1} \leq k_{2} \leq+\infty$.

Definition 1 [21] A collection of graphs $\{G(1), \ldots, G(m)\}$ is jointly connected if $\left(\mathcal{N}_{G}, \cup_{k=1}^{m} \mathcal{E}_{G}(k)\right)$ is a strongly connected graph. The agents are linked together across the interval $[l, m]$ if the collection of graphs $\{G(k), k \in[l, m]\}$ is jointly connected. A node $i$ is connected to all other nodes across a time set $\mathcal{T} \subseteq \mathbb{N}$ if $i$ is connected to all other nodes in the $\operatorname{graph}\left(\mathcal{N}_{G}, \bigcup_{k \in \mathcal{T}} \mathcal{E}_{G}(k)\right)$.

If $(j, i) \in \mathcal{E}_{G}$ we say that $j$ is neighbor to $i$ and the $j$-th agent transmits instantaneously its state to the $i$-th agent. The set of neighbors to the node $i \in \mathcal{N}_{G}$ is $\mathcal{N}_{i}(G)=\left\{j \in \mathcal{N}_{G}\right.$ : $\left.(j, i) \in \mathcal{E}_{G}\right\}$ and $\left|\mathcal{N}_{i}\right|$ is the valency of the $i$-th node.

We define the communication matrix $\tilde{K}(G)=\left[\tilde{k}_{i j}\right]_{i, j \in \mathcal{N}_{G}}$, $\tilde{K}(G) \in \mathbb{R}^{n \times n}$, where:

$$
\tilde{k}_{i j}= \begin{cases}\frac{w_{j i}}{1+\sum_{h \in \mathcal{N}_{i}(G)} w_{h i}} & \text { if } j \in \mathcal{N}_{i}(G), \\ \frac{1}{1+\sum_{h \in \mathcal{N}_{i}(G)} w_{h i}} & \text { if } i=j, \\ 0 & \text { otherwise. }\end{cases}
$$

$\tilde{K}(G)$ is a stochastic matrix (i.e. it is square and nonnegative and its row sums are equal to 1 , see [21]), whose entry $(i, j)$ is non null if and only if $i=j$ or $(j, i) \in \mathcal{E}_{G}$. We also define $K(G)=\tilde{K}(G) \otimes I_{d}$ where $\otimes$ denotes the Kronecker product and $I_{d}$ is the identity matrix of order $d$. Moreover we denote by $K_{i}(G) \in \mathbb{R}^{d \times d n}$ the $i$-th block of the matrix

\footnotetext{
${ }^{1}$ As remarked in [3], weights $w_{i j}$ and $w_{j i}$ can be different. If they are equal, one recovers the notion of undirected graphs.
}

$K(G)$, partitioned as $K(G)=\left[K_{1}^{T}(G) \cdots K_{n}^{T}(G)\right]^{T}$. Note that if $x(k)=\left[x_{1}^{T}(k) \cdots x_{n}^{T}(k)\right]^{T}$, with $x_{i}(k) \in \mathbb{R}^{d}$, one has

$$
\begin{aligned}
& K_{i}(G(k)) x(k) \in \mathcal{C}_{i}(G(k))= \\
& \operatorname{Co}\left(\left\{x_{i}(k)\right\} \cup\left\{x_{j}(k), j \in \mathcal{N}_{i}(G(k))\right\}\right)
\end{aligned}
$$

where $\operatorname{Co}(A)$ is the convex hull of the set $A$ (see [21]).

For sake of completeness, next we summarize some results provided in [3] that will enable us to prove consensus under the MPC schemes we will propose in the sequel. Assume that agents obey the general closed-loop dynamics

$$
x(k+1)=f(k, x(k))
$$

where $x(k)=\left[x_{1}(k)^{T} \cdots x_{n}(k)^{T}\right]^{T}$ and $x_{i}(k) \in \mathbb{R}^{d}, \forall i \in$ $\mathcal{N}_{G}$. The nodes of the network have reached consensus if and only if $x_{i}=x_{j}, \forall i, j \in \mathcal{N}_{G}, i \neq j$. The corresponding state value is called consensus point. The consensus subspace is $\Phi_{c}=\left\{x \in \mathbb{R}^{n d}: x_{1}=x_{2}=\cdots=x_{n}\right\}$.

Definition 2 [3] Let $\Phi \subseteq \mathbb{R}^{d n}$ be a set of equilibria for (3). System (3) is globally attractive w.r.t. $\Phi$ if for each $\phi_{1} \in \Phi$, $\forall c_{1}, c_{2}>0$ and $\forall k_{0} \in \mathbb{N}, \exists T \geq 0$ such that every solution $\zeta$ to (3) has the following property:

$\left\|\zeta\left(k_{0}\right)-\phi_{1}\right\|<c_{1} \Rightarrow \exists \phi_{2} \in \Phi:\left\|\zeta(k)-\phi_{2}\right\|<c_{2}, \forall k \geq k_{0}+T$

where $\|\cdot\|$ denotes the Euclidean norm. The system is uniformly globally attractive w.r.t. $\Phi$ if it is globally attractive w.r.t. $\Phi$ and the constant $T$ is independent of $k_{0}$.

Note that, as described in [3], global attractivity implies that all solutions to (3) converge to a point in $\Phi$ as $k \rightarrow+\infty$. However, the vice-versa is not true.

Definition 3 Assume that the set of equilibria associated to the multiagent system (3) is $\Phi_{c}$. Then, consensus is asymptotically reached if one of the following conditions holds:

1) $\mathcal{G}$ is a collection of directed graphs and (3) is uniformly globally attractive w.r.t. $\Phi_{c}$;

2) $\mathcal{G}$ is a collection of bidirectional graphs and (3) is globally attractive w.r.t. $\Phi_{c}$,

The consensus results stated in [3] hinge on the following assumption.

Assumption 1 For every graph $G(k) \in \mathcal{G}$, agent $i \in \mathcal{N}_{G}$ and state $x \in X^{n}, X \subseteq \mathbb{R}^{d}$, there is a compact set $e_{i}(G(k))(x) \subseteq$ $X$ such that:

1) $f_{i}(x, k) \in e_{i}(G(k))(x), \forall k \in \mathbb{N}, \forall x \in X^{n}$;

2) $e_{i}(G(k))(x)=\left\{x_{i}\right\}$ if $x_{i}=x_{j}, \forall j \in \mathcal{N}_{i}(G(k))$;

3) whenever the states of agent $i$ and agents $j \in \mathcal{N}_{i}(G(k))$ are not all equal, $e_{i}(G(k))(x) \in \operatorname{Ri}\left(\mathcal{C}_{i}(G(k))\right)$, where $\operatorname{Ri}(A)$ denotes the relative interior of the set $A$;

4) the set-valued function $e_{i}(G(k))(x): X^{n} \mapsto 2^{X}$ is continuous $\left(2^{X}\right.$ is the power set of $\left.X\right)$.

The main theorem on consensus we will use is stated next and exploits the following assumptions. 
Assumption 2 For the collection $\mathcal{G}$ of directed graphs there exists a non-negative integer $T \geq 0$ such that, $\forall k_{0} \in \mathbb{N}$, there is a node connected to all other nodes across $\left[k_{0}, k_{0}+T\right]$.

Assumption 3 For the collection $\mathcal{G}$ of bidirectional graphs and for all $k_{0} \in \mathbb{N}$, all agents are linked together across the interval $\left[k_{0},+\infty\right)$.

Theorem 1 [3] Let $\mathcal{G}$ be a collection of directed [resp. bidirectional] graphs and assume that $f$ in (3) verifies Assumption 1. Then, system (3) asymptotically reaches consensus if and only if Assumption 2 [resp. Assumption 3] holds.

For further comments and examples clarifying the minimality of the assumptions of Theorem 1 as well as the links between uniform global attractiveness [resp. global attractiveness] and directed [resp. bidirectional] graphs, we defer the reader to [3].

\section{MPC FOR CONSENSUS AMONG AGENTS WITH A SINGLE-INTEGRATOR DYNAMICS}

In this section we consider a system of $n$ agents, each one described by the following discrete-time single-integrator model

$$
x_{i}(k+1)=x_{i}(k)+u_{i}(k), \quad i \in \mathcal{N}_{G}
$$

with initial condition $x_{i}(0)=x_{i 0}$. The vector $u_{i}(k) \in \mathbb{R}^{d}$ is control input of agent $i$ at time $k$.

As an example, considering a group of autonomous vehicles moving in a $d$-dimensional geometric space, $x_{i}(k)$ describes the position of agent $i$. We will also assume that the communication topology is time-varying and captured by the collection of graphs $\mathcal{G}$.

Given a generic vector-valued signal $y(\cdot)$ and a positive integer $N$, let us denote by the capitalized vector $Y(k)=$ $\left[y^{T}(k), \cdots, y^{T}(k+N-1)\right]^{T}$ the values of $y(\cdot)$ over the time interval $[k, k+N-1]$.

Let $N \geq 1$ denote the length of the prediction horizon. We associate to the $i$-th agent the input sequence $U_{i}(k)$ and the cost

$$
\begin{aligned}
J_{i}\left(x(k), U_{i}(k)\right) & =J_{i}^{x}\left(x(k), U_{i}(k)\right)+J_{i}^{u}\left(U_{i}(k)\right) \\
J_{i}^{x}\left(x(k), U_{i}(k)\right) & =\alpha_{i} \sum_{j=1}^{N}\left\|x_{i}(k+j)-z_{i}(k)\right\|^{2} \\
J_{i}^{u}\left(U_{i}(k)\right) & =\beta_{i} \sum_{j=0}^{N-1}\left\|u_{i}(k+j)\right\|^{2}
\end{aligned}
$$

where

$$
z_{i}(k)=K_{i}(G(k)) x(k)
$$

defines the target point for the $i$-th agent and $\alpha_{i}, \beta_{i}>0$ are weights. Note that from (2), one has $z_{i}(k) \in \mathcal{C}_{i}(G(k))$ and, in particular, $z_{i}(k)$ is just the barycenter of $\left\{x_{i}(k)\right\} \bigcup\left\{x_{j}(k), j \in\right.$ $\left.\mathcal{N}_{i}(k)\right\}$ when the graph is unweighted.

Cost (5) is decentralized, because the term $z_{i}(k)$ depends only on the states of neighbors to the $i$-th agent at time $k$.
Consider the following Constrained Finite-Time Optimal Control (CFTOC) problem for agent $i \in \mathcal{N}_{G}$ :

$$
\min _{U_{i}(k)} J_{i}\left(x(k), U_{i}(k)\right)
$$

subject to the following constraints:

(a) the agent dynamics (4);

(b) the input constraint

$$
\left\|u_{i}(k+j)\right\| \leq u_{i, \max },
$$

with $u_{i, \max }>0, j \in[0, N-1]$.

Optimal inputs computed at time $k$ will be denoted with $U_{i}^{o}(k \mid k)$ and we will investigate the consensus properties provided by the receding-horizon control law

$$
u_{i}^{R H}(k)=\kappa_{i}^{R H}\left(k, z_{i}(k)\right), \quad \kappa_{i}^{R H}\left(k, z_{i}(k)\right)=u_{i}^{o}(k \mid k)
$$

Note that $u_{i}^{R H}(k)$ coincides with the first input sample appearing in the vector $U_{i}^{o}(k \mid k)=$ $\left[u_{i}^{o T}(k \mid k), u_{i}^{o T}(k+1 \mid k), \cdots, u_{i}^{o T}(k+N-1 \mid k)\right]^{T} . \quad$ Note also that the state-feedback $\kappa_{i}^{R H}$ is time-varying when the communication network changes over time. Problem (9) is always feasible since $U_{i}(k)=0$ is a feasible input sequence. We are now in a position to state the main result of this Section. Its proof is based on the application of Theorem 1 and the geometrical properties of discrete-time paths introduced in the Appendix.

Theorem 2 Let $\mathcal{G}$ be a sequence of directed [resp. bidirectional] graphs. The closed-loop multi-agent system given by (4) and (11) asymptotically reaches consensus if and only if Assumption 2 [resp. Assumption 3] holds.

Proof: Consider the closed-loop multi-agent system corresponding to equations (4) and (11), that can be represented by

$$
x(k+1)=f(x(k)),
$$

where

$f(x(k))=\left[\begin{array}{c}f_{1}(x(k)) \\ \vdots \\ f_{n}(x(k))\end{array}\right], f_{i}(x(k))=x_{i}(k)+\kappa_{i}^{R H}\left(k, z_{i}(k)\right)$

Apparently, from (9), (10) and (11) one has $\kappa_{i}^{R H}\left(k, z_{i}(k)\right)=$ $0, \forall i \in \mathcal{N}_{G}$ if and only if $x(k) \in \Phi_{c}$. Hence, $\Phi_{c}$ collects the consensus equilibria of (12). Next, we show that the map $f$ fulfills Assumption 1.

With the notations used in Assumption 1 , we set $X=\mathbb{R}^{d}$ and $e_{i}(G(k))(x)=\left\{f_{i}(x)\right\}$. Then point 1 of Assumption 1 is trivially verified. For point 2 , when $x_{i}(k)=x_{j}(k), \forall j \in$ $\mathcal{N}_{i}(G(k))$, then $z_{i}(k)=x_{i}(k)$. From the definition of the cost $J_{i}$, it is immediate to verify that $J_{i}(x(k), 0)=0$ and hence the optimal inputs $U_{i}^{o}(x(k))$ are zero. This implies that $f_{i}(x)=x_{i}$, and point 2 is verified.

The core part of the proof consists in verifying point 3 . To this purpose we will use the tools and the terminology for paths described in the Appendix.

If there exists $j \in \mathcal{N}_{i}(G(k))$ such that $x_{i}(k) \neq x_{j}(k)$ one has $x_{i}(k) \neq z_{i}(k)$. Let $X_{i}\left(x(k), U_{i}(k)\right)$ be the sequence of 
states of the $i$-th agent generated by the initial state $x(k)$ and the input sequence $U_{i}(k)$, with $u_{i}(k+j), j \in[0, N-1]$ fulfilling the constraint (10).

As a first step we show that $X_{i}^{o}\left(x(k), U_{i}^{o}(k)\right)=$ $\left[x_{i}^{o T}(k) \cdots x_{i}^{o T}(k+N)\right]^{T}$ is a path pointing towards $z_{i}(k)$. Note that, because of the single-integrator dynamics (4), the term $J_{i}^{u}$ can be rewritten as:

$$
J_{i}^{u}(k)=\beta_{i} \sum_{j=0}^{N-1}\left\|x_{i}(k+j+1)-x_{i}(k+j)\right\|^{2}
$$

Assume by contradiction that $X_{i}^{o}\left(x(k), U_{i}^{o}(k)\right)$ is not pointing towards $z_{i}(k)$. Theorem 5 (see the Appendix) shows that there is an $N$-path $\hat{X}_{i}(k)=\left[\hat{x}_{i}^{T}(k) \cdots \hat{x}_{i}^{T}(k+N)\right]^{T}$ with $\hat{x}_{i}(k)=$ $x_{i}^{o}(k)$ pointing towards $z_{i}(k)$ and such that, $\forall j=0, \ldots,(N-$ $1)$, both the following inequalities hold simultaneously:

$$
\begin{array}{r}
\left\|\hat{x}_{i}(k+j+1)-z_{i}(k)\right\| \leq\left\|x_{i}^{o}(k+j+1)-z_{i}(k)\right\| \\
\left\|\hat{x}_{i}(k+j+1)-\hat{x}_{i}(k+j)\right\| \leq\left\|x_{i}^{o}(k+j+1)-x_{i}^{o}(k+j)\right\|
\end{array}
$$

Inequality (15) implies that the input vector $\hat{U}_{i}(k)$, producing $\hat{X}_{i}(x(k))$, fulfills the constraint (10). Moreover (14) implies that $J_{i}^{x}\left(x(k), \hat{U}_{i}(k)\right) \leq J_{i}^{x}\left(x(k), U_{i}^{o}(k)\right)$ and (15) that $J_{i}^{u}\left(\hat{U}_{i}(k)\right) \leq J_{i}^{u}\left(U_{i}^{o}(k)\right)$. Hence $X_{i}^{o}\left(x(k), U_{i}^{o}(k)\right)$ is not optimal. One therefore concludes that the optimal state path $X_{i}^{o}\left(x(k), U_{i}^{o}(k)\right)$ is necessarily pointing towards $z_{i}(k)$.

As a second step, we show that $x_{i}(k) \neq z_{i}(k)$ implies that $u_{i}^{o}(k) \neq 0$. This will be proved by first showing, by contradiction, that $U_{i}^{o}(x(k)) \neq 0$. Assume that $U_{i}^{o}(x(k))=$ 0 is the optimal input sequence. Then $J_{i}\left(x(k), U_{i}^{o}(k)\right)=$ $N \alpha_{i}\left\|x_{i}(k)-z_{i}(k)\right\|^{2}$. Consider the input sequence $\bar{U}_{i}(k)=$ $\left[\delta\left(z_{i}(k)-x_{i}(k)\right)^{T} 0^{T} \cdots 0^{T}\right]^{T}, \quad 0<\delta \leq 1$, where $\delta$ is such that $\left\|\delta\left(z_{i}(k)-x_{i}(k)\right)\right\| \leq u_{i, \max }$, and let $\bar{X}(k)=$ $[\bar{x}(k) \cdots \bar{x}(k+N)]$ be the corresponding state path. One has

$$
\begin{aligned}
J_{i}\left(x(k), \bar{U}_{i}(k)\right)= & N \alpha_{i}(1-\delta)^{2}\left\|x_{i}(k)-z_{i}(k)\right\|^{2} \\
& +\beta_{i} \delta^{2}\left\|x_{i}(k)-z_{i}(k)\right\|^{2}
\end{aligned}
$$

For $\delta<\frac{2 N \alpha_{i}}{N \alpha_{i}+\beta_{i}}$ one has $J\left(x(k), \bar{U}_{i}(k)\right)<J\left(x(k), U_{i}^{o}(k)\right)$. Such a choice is always feasible since $\frac{2 N \alpha_{i}}{N \alpha_{i}+\beta_{i}}>0$. Therefore $U_{i}^{o}(k)=0$ cannot be the optimal input sequence.

Now we prove that $u_{i}^{o}(k) \neq 0$ by contradiction. Assume that the optimal input sequence is $U_{i}^{o}(k)=\left[\begin{array}{ll}0^{T} & u_{i}^{o T}(k+\end{array}\right.$ 1) $\left.\cdots u_{i}^{o T}(k+N-1)\right]^{T}$, with $u_{i}^{o T}(k+1) \neq 0$. Now consider the input $\bar{U}_{i}(k)=\left[u_{i}^{o T}(k+1) \cdots u_{i}^{o T}(k+N-1) 0^{T}\right]^{T}$. We observe that all input samples in $\bar{U}_{i}(k)$ verify the constraint (10), as those of $U_{i}^{o}(k)$ do. Then,

$$
\begin{aligned}
& J\left(x(k), \bar{U}_{i}(k)\right)-J\left(x(k), U_{i}^{o}(k)\right) \\
& =\alpha_{i}\left\|x_{i}(k)+\sum_{j=0}^{N-1} u_{i}^{o}(k+j)-z_{i}(k)\right\|^{2} \\
& \quad-\alpha_{i}\left\|x_{i}(k)-z_{i}(k)\right\|^{2}<0
\end{aligned}
$$

Inequality (17) follows from the fact that some input $u_{i}^{o}(k+$ $j), j=0, \ldots,(N-1)$ is non null and every input makes the state follow a path that points towards $z_{i}(k)$.

The property of stochasticity of the matrix $K(G(k))$, together with the assumptions that $\mathcal{N}_{i}(G) \neq \emptyset$ and $x_{i}(k) \neq z_{i}(k)$ implies that $z_{i}(k) \in \operatorname{Ri}\left(\mathcal{C}_{i}(G(k))\right)$. When also $x_{i}(k) \in$ $\operatorname{Ri}\left(\mathcal{C}_{i}(G(k))\right)$ it is trivial to conclude that the optimal trajectory belongs to $\operatorname{Ri}\left(\mathcal{C}_{i}(G(k))\right)$. When $x_{i}(k)$ belongs to the boundary of $\mathcal{C}_{i}(G(k))$, by applying the line segment principle [22] the same conclusion follows because the optimal trajectory is totally included in the relatively open segment connecting $x_{i}(k)$ and $z_{i}(k)$.

Finally, also point 4 in Assumption 1 is verified. In fact, since we have proved that the optimal state trajectory $X_{i}^{o}\left(x_{i}(k), U_{i}^{o}(k)\right)$ is pointing towards $z_{i}(k)$, it is possible to write: $x_{i}(k+1)=x_{i}(k)+\eta(k) L(k)\left(z_{i}(k)-x_{i}(k)\right), \forall k \in \mathbb{N}$, where $0 \leq L(k) \leq 1$ (specifically, $L(k)=0$ only when $\left.z_{i}(k)=x_{i}(k)\right)$ and

$$
\eta(k)= \begin{cases}1 & \text { if } L(k)\left\|z_{i}(k)-x_{i}(k)\right\| \leq u_{i, \max } \\ \frac{u_{i, \max }}{L(k)\left\|z_{i}(k)-x_{i}(k)\right\|} & \text { otherwise }\end{cases}
$$

It is then apparent that the function $f_{i}(x)$ is continuous, and therefore $e_{i}(G(k))(x)$ is also continuous.

In conclusion, Assumption 1 holds and the rest of the proof is a straightforward application of Theorem 1 .

Remark 1 By suitably choosing the weights $\alpha_{i}$ and $\beta_{i}$ in (6) and (7), respectively, it is possible to tune the behavior of each agent. For instance, given an unweighted or bidirectional graph, it is possible to mimic behaviors of the agents typically obtained by using weighted or directed graphs. This feature is illustrated in Example 1 where it is shown how to achieve, approximately, leader-following.

Example 1 We consider a set of $n=5$ agents moving in a two dimensional space, with initial states $x_{1}(0)=\left[\begin{array}{ll}-30 & 30\end{array}\right]^{T}$, $x_{2}(0)=\left[\begin{array}{ll}-25 & 35\end{array}\right]^{T}, x_{3}(0)=\left[\begin{array}{c}65-75\end{array}\right]^{T}, x_{4}(0)=[70-68]^{T}$, $x_{5}(0)=[100-25]^{T}$. The prediction horizon is $N=3$. The weights in the cost function (5) are $\alpha_{i}=1, i=1, \ldots, 5$, $\beta_{1}=100, \beta_{i}=1, i=2, \ldots, 5$. The communication network is described by the time-invariant unweighted bidirectional graph represented in Fig. 1, that corresponds to the following communication matrix:

$$
\tilde{K}(G)=\left(\begin{array}{ccccc}
\frac{1}{2} & \frac{1}{2} & 0 & 0 & 0 \\
\frac{1}{3} & \frac{1}{3} & \frac{1}{3} & 0 & 0 \\
0 & \frac{1}{3} & \frac{1}{3} & \frac{1}{3} & 0 \\
0 & 0 & \frac{1}{3} & \frac{1}{3} & \frac{1}{3} \\
0 & 0 & 0 & \frac{1}{2} & \frac{1}{2}
\end{array}\right)
$$

The input constraints (10) are given by $u_{i, \max }=100, i=$

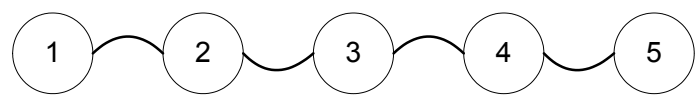

Fig. 1. Communication network used in Examples 1, 2 and 3

$1, \ldots, 5$. The simulation in Fig. 2 shows asymptotic convergence towards a consensus point. Since $\beta_{1} \gg \beta_{i}, i=$ $2,3,4,5$, agent 1 behaves approximately as a leader, hence moving much less than the others from its initial position. In this case, input constraints are never active. It is important to notice that in this example both cost functions $J_{1}\left(x^{o}(k), U_{1}^{o}(k \mid k)\right)$ and $J^{o}\left(x^{o}(k), U^{o}(k \mid k)\right)=$ 

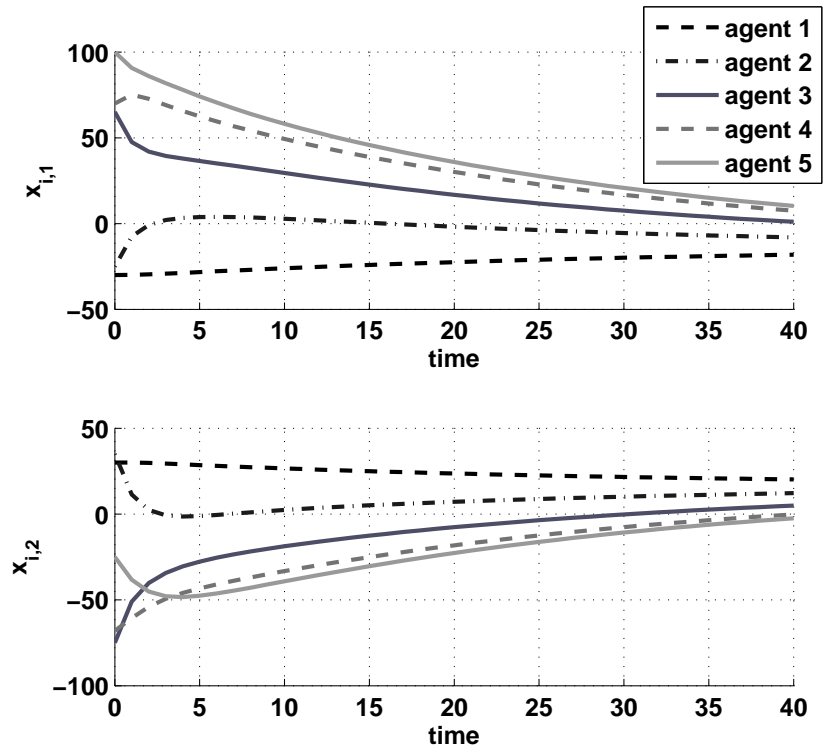

Fig. 2. Example 1: Evolution of agents' state.

$\sum_{i=1}^{5} J_{i}\left(x^{o}(k), U_{i}^{o}(k \mid k)\right)$, where $x^{0}(k)$ is the state sequence given by (4) and (11), are not monotonically decreasing over time (see Fig. 3). This implies that the global cost function $J^{o}(x(k))$ cannot be used as a Lyapunov function and classical arguments for proving the stability of MPC algorithms (see e.g. [18], [19]) cannot be adopted. This justifies the necessity of an alternative technique to prove convergence, as the one we propose, based on geometrical concepts and on Theorem 1.

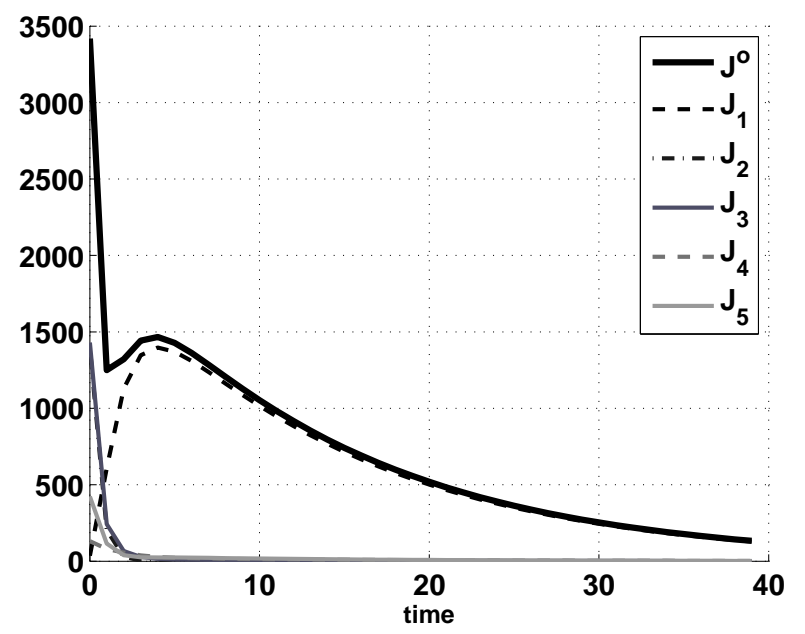

Fig. 3. Example 1: the bold line represents the global cost $J^{o}\left(x^{o}(k), U^{o}(k \mid k)\right)$. The other lines represent the cost of individual agents.

Example 2 We use the same setting of Example 1, but we now choose $\beta_{i}=1, i=1, \ldots, 5$ and $u_{i, \max }=5$. We also assume that the communication network is time-varying and fulfills Assumption 3. More in detail, we generated a random sequence of unweighted bidirectional graphs such that all agents are linked together across the interval $\left[k_{0},+\infty\right), \forall k_{0} \in \mathbb{N}$. As seen in Fig. 4, consensus is asymptotically achieved, in accordance with Theorem 2, even though input constraints are active at times $k \leq 10$, as shown in Fig. 5. Notice that the value of
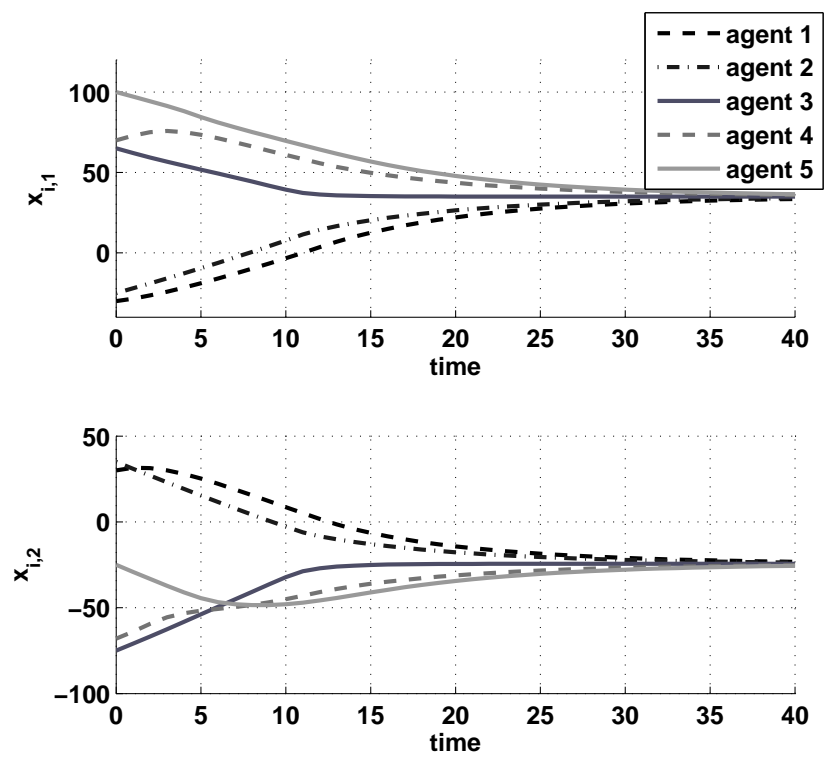

Fig. 4. Example 2: Evolution of agents' state.
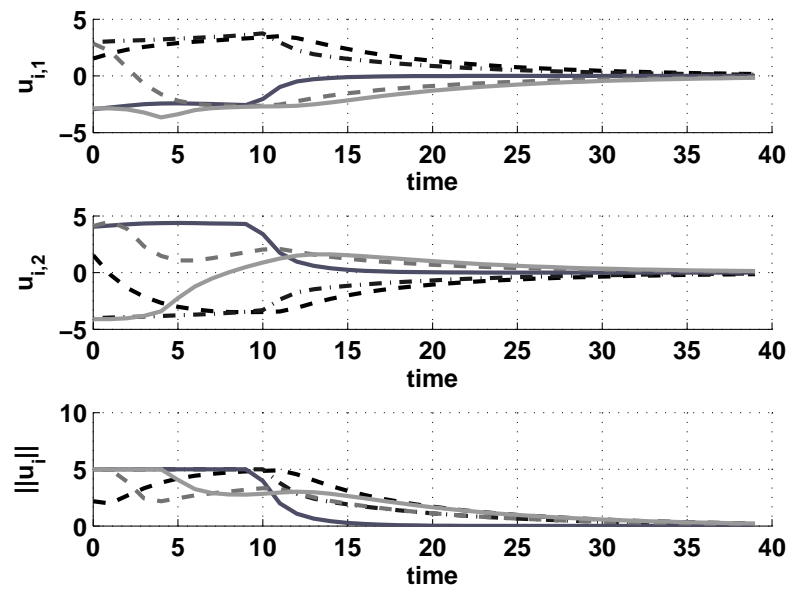

Fig. 5. Example 2: Control inputs of individual agents.

the asymptotic consensus point in this example differs from the one in the previous one, though the initial conditions of the system are the same in both cases.

\section{A CONTRACTIVE MPC SCHEME FOR AGENTS WITH SINGLE-INTEGRATOR DYNAMICS}

The CFTOC problem (9) does not include constraints on the agents' state. We now introduce an alternative MPC law requiring periodic communication among agents and using specific state constraints in order to guarantee consensus. This solution has the advantage that it can be easily extended to 
agents with double-integrator dynamics (see Section V). The control scheme is inspired by the MPC technique proposed in [20], where a "contractive" constraint is imposed on the state to guarantee stability.

For the $i$-th agent, whose dynamics is described by (4), consider the following $\operatorname{cost}^{2}$ :

$$
\begin{aligned}
& J_{i}\left(x_{i}(k), z_{i}(p(k) N), U_{i}(k)\right)= \\
& \quad J_{i}^{x}\left(x_{i}(k), z_{i}(p(k) N), U_{i}(k)\right)+J_{i}^{u}\left(U_{i}(k)\right)
\end{aligned}
$$

where

$$
\begin{aligned}
& J_{i}^{x}\left(x_{i}(k), z_{i}(p(k) N), U_{i}(k)\right)= \\
& \alpha_{i} \sum_{j=1}^{N}\left\|x_{i}(k+j)-z_{i}(p(k) N)\right\|^{2}
\end{aligned}
$$

and $J_{i}^{u}$ is defined as in (7). The function $p(\cdot)$ is defined as

$$
p(k)=\left\lfloor\frac{k}{N}\right\rfloor
$$

where $\lfloor\xi\rfloor$ denotes the greatest integer lower bound to $\xi \in \mathbb{R}$; a representation of $p(k)$ is provided in Fig. 6 for $N=4$.

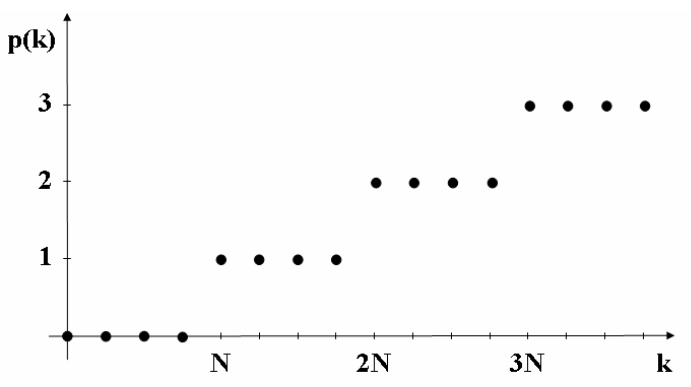

Fig. 6. Function $p(k)$ for $N=4$.

We associate to $k \in \mathbb{N}$ the p-interval $P(k)=$ $[p(k) N,(p(k)+1) N-1]$. Note that $p(k)$ is constant over each $p$-interval.

Consider the following CFTOC problem for agent $i \in \mathcal{N}_{G}$ :

$$
\min _{U_{i}(k)} J_{i}\left(x_{i}(k), z_{i}(p(k) N), U_{i}(k)\right)
$$

subject to the following constraints:

(A) the agent dynamics (4);

(B) the input constraint

$$
\left\|u_{i}(k+j)\right\| \leq u_{i, \max },
$$

with $u_{i, \max }>0, i \in \mathcal{N}_{G}, j \in[0, N-1]$;

(C) the state constraint

$$
x_{i}((p(k)+1) N)=z_{i}(p(k) N) .
$$

where the target point $z_{i}(k)$ has been defined in (8).

The corresponding receding-horizon control law will be denoted as

$$
\begin{aligned}
& u_{i}^{R H}(k)=\kappa_{i}^{R H}\left(k, x_{i}(k), z_{i}(p(k) N)\right), \\
& \quad \kappa_{i}^{R H}\left(k, x_{i}(k), z_{i}(p(k) N)\right)=u_{i}^{o}(k \mid k)
\end{aligned}
$$

\footnotetext{
${ }^{2}$ With a little abuse of notation we use here the same symbol $J_{i}$ appearing in $(5)$
}

Constraint (24) is such that:

- it is defined on the multi-agent system state at the first time instant after the end of $P(k)$;

- it changes when the current time $k$ switches from a $p$ interval to the next one;

- it is the same for all $k \in P(k)$; consequently, while $k$ approaches the end of a $p$-interval, the difference between the next time at which the state is constrained and $k$ decreases.

Equation (24) can be interpreted as a "contractive" constraint because, under suitable conditions on the communication network, it forces the shrinking of the convex hull spanned by the agents' state, as it will be shown in the sequel. Note that when $k=N l$, (24) is also a terminal constraint. Obviously the fact that this constraint can be fulfilled or not depends on the kind of application we consider.

Remark 2 For $l \in \mathbb{N}$ and $k \in[l N+1,(l+1) N-1]$ the cost (19) is independent of the graph $G(k)$ and the states $x_{i}(k), i \in \mathcal{N}_{i}(k)$. This implies that agents are required to transmit their state to neighbors only at times $l N, l \in \mathbb{N}$.

Remark 3 Note that if at times $l N, l \in \mathbb{N}$ the CFTOC problem (22) is feasible, then it is feasible also at times $l N+j, j \in[1, N-1]$. Indeed let $X_{i}^{o}\left(x(l N), U_{i}^{o}(l N \mid l N)\right)$ be the sequence of states produced by $x_{i}(l N)$ and $U_{i}^{o}(l N \mid l N)$. $U_{i}^{o}(l N \mid l N)$ steers $x_{i}(l N)$ into $x_{i}(l N+N)=z_{i}(l N)$ in order to fulfill constraint $(C)$. Hence, at time $k=l N+j$ the sequence

$$
U_{i}(l N+j)=\left[\begin{array}{c}
u_{i}^{o}(l N+j \mid l N) \\
\vdots \\
u_{i}^{o}(l N+N-1 \mid l N) \\
0 \\
\vdots \\
0
\end{array}\right]
$$

is feasible (since it steers $x_{i}^{o}(l N+j)$ into $x_{i}^{o}((l+1) N)=$ $\left.z_{i}(l N)\right)$ and optimal (by Bellman's principle, see [23]). This means that in the nominal case, problem (22) needs to be solved just at times $l N, l \in \mathbb{N}$. However, resorting to a receding-horizon strategy allows one to apply at any time a state-feedback control law.

The following result shows that an appropriate choice of the prediction horizon $N$ always ensures the feasibility of the control problem.

Lemma 1 The CFTOC problem (22) with constraints (A), (B), (C) is feasible at all times if $N \geq \max _{i \in \mathcal{N}_{G}} N_{i}$ where

$$
N_{i}=\left\lceil\frac{\max _{j \in \mathcal{N}_{G}}\left\|x_{i}(0)-x_{j}(0)\right\|}{u_{i, \max }}\right\rceil
$$

and $\lceil\xi\rceil$ denotes the least integer upper bound to $\xi \in \mathbb{R}^{+}$.

Proof: In view of Remark 3, we focus only on feasibility at time instants $l N$. Because of the integrator dynamics (4), at time $k=0$ agent $i$ can reach in $\tilde{N}_{i}$ steps all states $\bar{x}_{i} \in \mathbb{R}^{d}$ 
verifying $\left\|\bar{x}_{i}-x_{i}(0)\right\| \leq \tilde{N}_{i} u_{i, \max }$. Therefore, constraint (C) with $k=0$ can be met if an horizon $\tilde{N}$ is chosen, where

$$
\begin{gathered}
\tilde{N} \geq \max _{i \in \mathcal{N}_{G}} \tilde{N}_{i} \\
\tilde{N}_{i}=\left\lceil\frac{\left\|z_{i}(0)-x_{i}(0)\right\|}{u_{i, \max }}\right\rceil
\end{gathered}
$$

Using the fact that $K_{i}(G(0)) x(0) \in \mathcal{C}_{i}(G(0))$, one has

$$
\left\|z_{i}(0)-x_{i}(0)\right\| \leq \max _{\forall i, j \in \mathcal{N}_{G}}\left\|x_{i}(0)-x_{j}(0)\right\|
$$

and therefore, $N_{i} \geq \tilde{N}_{i}, \forall i \in \mathcal{N}_{G}$ and $N \geq \tilde{N}$. Hence, feasibility at time $k=0$ holds. Let now $l \geq 1$. Using the same rationale, the CFTOC problem (22) is feasible if an horizon $\tilde{N}_{l}$ is chosen where $\tilde{N}_{l} \geq \max _{i \in \mathcal{N}_{G}} \tilde{N}_{i, l}$ and

$$
\tilde{N}_{i, l}=\left\lceil\frac{\left\|z_{i}(l N)-x_{i}(l N)\right\|}{u_{i, \max }}\right\rceil
$$

Note that $x(l N)$ is the state produced by the optimal inputs and then $x_{i}(l N)=K_{i}(G((l-1) N)) x((l-1) N)$. It is easy to prove that, $\forall i \in \mathcal{N}_{G}, x_{i}(l N) \in \mathcal{C}_{i}(G((l-1) N))$ and proceeding backwards, one has $x_{i}(l N) \in X_{0}$ and then $z_{i}(l N) \in \mathcal{C}_{i}(G(0))$. Hence,

$$
\left\|z_{i}(l N)-x_{i}(l N)\right\| \leq \max _{\forall i, j \in \mathcal{N}_{G}}\left\|x_{i}(0)-x_{j}(0)\right\|
$$

and therefore one has $N_{i} \geq \tilde{N}_{i, l}, \forall i \in \mathcal{N}_{G}$ and $N \geq \tilde{N}_{l}, \forall l \in$ $\mathbb{N}, l \geq 1$. This completes the proof.

We are now in a position to state the main results of this section.

Theorem 3 Let $\mathcal{F}=\{G(l N), l \in \mathbb{N}\}$ be a collection of directed [resp. bidirectional] graphs and assume that $N$ is such that the CFTOC problem (22) with constraints (A), (B) and (C) is feasible at all times. Then, the closed-loop multiagent system given by (4) and (25) asymptotically reaches consensus if and only if Assumption 2 [resp. Assumption 3] holds with $\mathcal{G}$ replaced by $\mathcal{F}$. Furthermore, denoting with $x_{i}^{o}(k)$ the optimal states, one has that

P.1 $x_{i}^{o}(k) \in \overline{x_{i}^{o}(p(k) N) z_{i}(p(k) N)}$, where $\overline{A B}$ is the line segment connecting points $A$ and $B$;

$P .2$ for all $l \in \mathbb{N}, d(j \mid l N)=\left\|z_{i}(l N)-x_{i}^{o}(l N+j)\right\|$ is a non-increasing function of $j \in[0, N)$.

Proof: For the sake of readability the proof is split in three parts.

Part 1. The contractive constraint (24) only depends on $K(G(\cdot))$ at times $l N \forall l \in \mathbb{N}$. It therefore holds

$$
x^{o}((l+1) N)=K(G(l N)) x^{o}(l N)
$$

It is simple to verify that system (33) fulfills Assumption 1. Therefore, Theorem 1 proves that the update map (33) is uniformly globally attractive [resp. globally attractive] with respect to $\Phi_{c}$ if and only if Assumption 2 [resp. Assumption $3]$ holds. If $N=1$, this completes the proof of the whole theorem.

Part 2. When $N>1$, we show properties (P.1) and (P.2) using the tools provided in the Appendix. First, note that, in view of Remark 3, (P.1) and (P.2) are equivalent to the fact that for all $l \in \mathbb{N}$ the optimal state path $X_{i}^{o}\left(x(l N), U_{i}^{o}(l N)\right)=$ $\left[x_{i}^{o T}(l N), \ldots, x_{i}^{o T}((l+1) N)\right]^{T}$ points towards $z_{i}(l N)$ (see Definition 8). To show this, one can use Theorem 5 , where $x_{i}^{o}(l N+j)=B_{j}, \forall k$ and $T_{A}$ is associated to a generic feasible state path. Note that, due to the contractive constraint (24), one has $A_{N}=B_{N}=0$.

Part 3. We finally show that when the components of $x^{o}(k)$ fulfill (P.1) then system (4) and (11) is uniformly globally attractive [resp. globally attractive] with respect to $\Phi_{c}$ if and only if (33) has the same property.

The "only if" part is obvious since $x^{o}(l N)$ is a subsequence of $x^{o}(k)$. For the "if" part we are assuming that

$$
\begin{aligned}
& \forall \tilde{\phi}_{1} \in \Phi_{c}, \forall \tilde{c}_{1}, \tilde{c}_{2}>0, \forall l_{0} \in \mathbb{N}, \exists L \geq 0: \\
& \left\|x^{o}\left(l_{0} N\right)-\tilde{\phi}_{1}\right\|<\tilde{c}_{1} \Rightarrow \exists \tilde{\phi}_{2} \in \Phi_{c}:\left\|x^{o}(l N)-\tilde{\phi}_{2}\right\|<\tilde{c}_{2}, \\
& \forall l \geq l_{0}+L .
\end{aligned}
$$

and the goal is to show that

$$
\begin{aligned}
& \forall \phi_{1} \in \Phi_{c}, \forall c_{1}, c_{2}>0, \forall k_{0} \in \mathbb{N}, \exists T \geq 0: \\
& \left\|x^{o}\left(k_{0}\right)-\phi_{1}\right\|<c_{1} \Rightarrow \exists \phi_{2} \in \Phi_{c}:\left\|x^{o}(k)-\phi_{2}\right\|<c_{2}, \\
& \forall k \geq k_{0}+T .
\end{aligned}
$$

Fix $\phi_{1}, c_{1}, c_{2}$ in (35). Using the triangle inequality, one obtains $\left\|x^{o}\left(l_{0} N\right)-\tilde{\phi}_{1}\right\| \leq \tilde{c}_{1}, \tilde{c}_{1}=\left\|x^{o}\left(l_{0} N\right)-x^{o}\left(k_{o}\right)\right\|+\left\|x^{o}\left(k_{o}\right)-\tilde{\phi}_{1}\right\|$ where we have chosen $l_{0}=p\left(k_{0}\right)$ and $\tilde{\phi}_{1}=\phi_{1}$. Set $\tilde{c}_{2}=\frac{c_{2}}{2}$. Formula (34) guarantees that

$$
\exists L \geq 0, \exists \tilde{\phi}_{2} \in \Phi_{c}:\left|x^{o}(l N)-\tilde{\phi}_{2}\right|<\tilde{c}_{2}, \forall l \geq l_{0}+L .
$$

Note that, in property (P.1), $z(p(k) N)=x^{o}((p(k)+1) N)$. Moreover (P.1) implies that

$$
\left\|x^{o}(k)-\tilde{\phi}_{2}\right\| \leq\left\|x^{o}(p(k) N)-\tilde{\phi}_{2}\right\|+\left\|x^{o}((p(k)+1) N)-\tilde{\phi}_{2}\right\|
$$

and hence, for all $k$ such that $p(k) \geq l_{0}+L$ (i.e. $k \geq\left(l_{0}+\right.$ $L) N$ ), formulae (36) and (37) yield $\left\|x^{o}(k)-\tilde{\phi}_{2}\right\|<2 \tilde{c}_{2}=c_{2}$. Therefore, (35) holds with $T=\left(l_{o}+L\right) N-k_{o}$ and $\phi_{2}=\tilde{\phi}_{2}$.

Remark 4 If there exists $k \in \mathbb{N}$ such that $G(p(k) N)$ is complete, consensus is reached at time $(p(k)+1) N$. In fact, in this case, $\forall i, j \in \mathcal{N}_{G}$

$$
K_{i}(G(p(k) N)) x(p(k) N)=K_{j}(G(p(k) N)) x(p(k) N)
$$

as it can be readily seen from the definition of the matrices $K_{i}(G)$. We also highlight that the consensus point, in the case of the contractive technique, does only depend on the set of communication graphs $\{G(p(k)), k \in \mathbb{N}\}$ (see [21] for the case of unweighted graphs).

Example 3 We apply the contractive MPC scheme using the same setting as in Example 1, with the exception that the prediction horizon is $N=10$. Simulations depicted in Figures 7-8 confirm the expected tendency of agents to consensus. Notice that, quite differently from Example 1, the control eliminates the quasi-"leader-follower" behavior. This is due 
to the presence of the contractive constraint, which forces agents to reach states which are not dependent on the weights $\alpha_{i}$ and $\beta_{i}$. Nevertheless, a leader-follower behavior could be approximated, also with a bidirectional graph, using suitably weighted graphs.
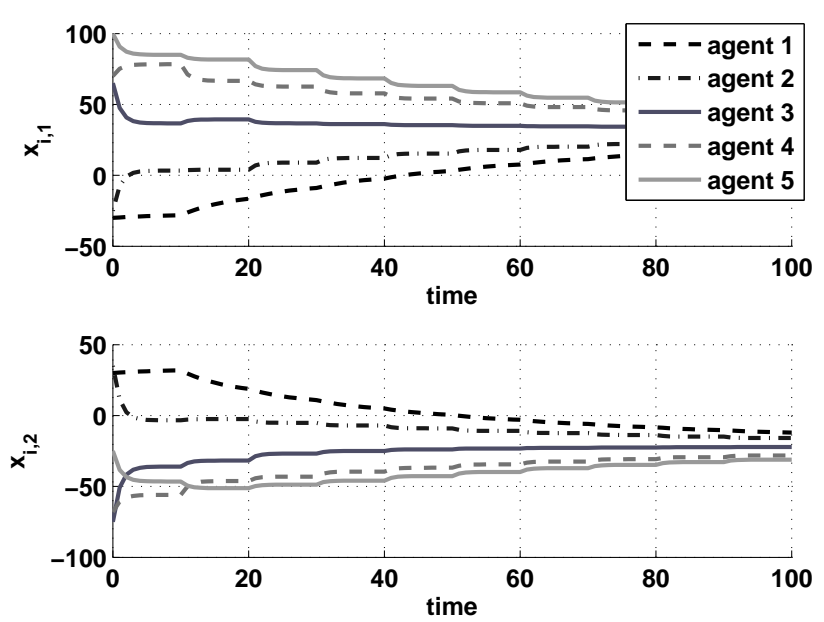

Fig. 7. Example 3: Evolution of agents' state.

In this example we assume $u_{i, \max }=\infty, \forall i$. The cost functions associated to each agent and the global cost function have sudden growths at the beginning of each p-interval, due to the changes in $K(G(p(\cdot) N))$.

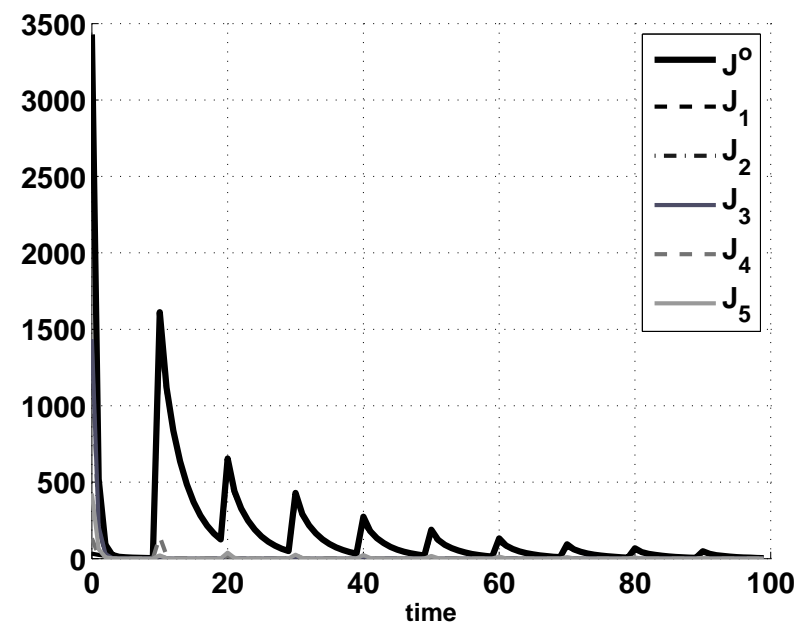

Fig. 8. Example 3: Optimal cost of individual agents $J_{i}\left(x^{o}(k), U_{i}^{o}(k \mid k)\right)$ and global optimal cost $J^{o}\left(x^{o}(k)\right)=\sum_{i=1}^{5} J_{i}\left(x^{o}(k), U_{i}^{o}(k \mid k)\right)$ (bold line).

Example 4 We consider the setting of Example 2, with the difference that here the prediction horizon is $N=5$ and $u_{i, \max }=20$. Moreover, the collection of graphs $\mathcal{F}$ is structured as follows:

$$
\mathcal{F}=\{\underbrace{G_{1}}_{1} G_{2} \underbrace{G_{1} G_{1}}_{2} G_{2} \underbrace{G_{1} G_{1} G_{1}}_{3} G_{2} \cdots\}
$$

where

$$
\begin{aligned}
& \tilde{K}\left(G_{1}\right)=\left(\begin{array}{ccccc}
\frac{1}{2} & \frac{1}{2} & 0 & 0 & 0 \\
\frac{1}{2} & \frac{1}{2} & 0 & 0 & 0 \\
0 & 0 & \frac{1}{2} & \frac{1}{2} & 0 \\
0 & 0 & 0 & \frac{1}{2} & \frac{1}{2} \\
0 & 0 & 0 & \frac{1}{2} & \frac{1}{2}
\end{array}\right) \\
& \tilde{K}\left(G_{2}\right)=\left(\begin{array}{cccccc}
\frac{1}{2} & \frac{1}{2} & 0 & 0 & 0 \\
\frac{1}{2} & \frac{1}{2} & 0 & 0 & 0 \\
0 & \frac{1}{2} & \frac{1}{2} & 0 & 0 \\
0 & 0 & \frac{1}{3} & \frac{1}{3} & \frac{1}{3} \\
0 & 0 & 0 & \frac{1}{2} & \frac{1}{2}
\end{array}\right)
\end{aligned}
$$

Note that $\mathcal{F}$ is a collection of unweighted directed graphs that does not fulfill the assumptions of Theorem 3. In fact, the communication graph $G_{1}$, which has no spanning tree, is active over time intervals of increasing length. Therefore asymptotic consensus is not achieved (see Fig. 9).
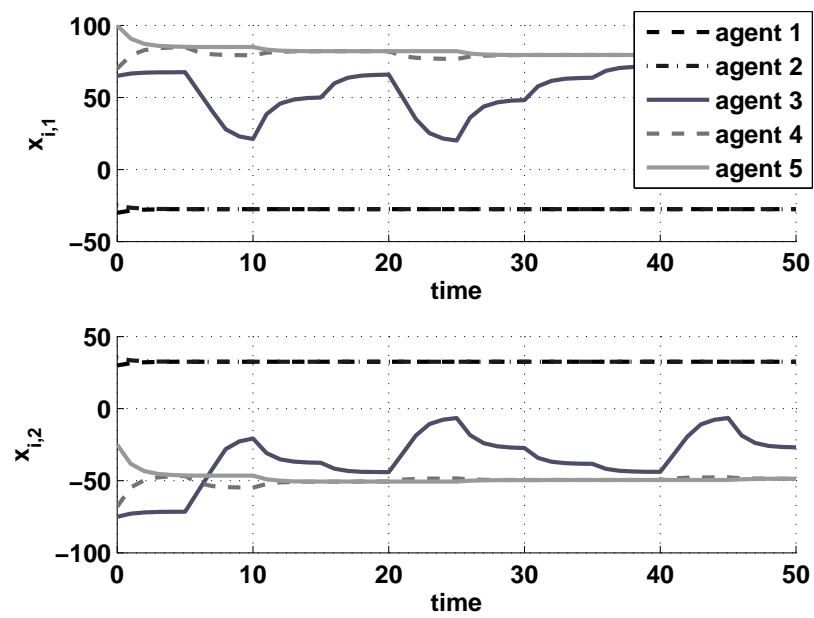

Fig. 9. Example 4: Evolution of agents' state.

\section{A CONTRACTIVE MPC SCHEME FOR AGENTS WITH DOUBLE-INTEGRATOR DYNAMICS}

We now consider a set of $n$ agents with discrete-time dynamics

$$
\begin{aligned}
& r_{i}(k+1)=r_{i}(k)+v_{i}(k) \\
& v_{i}(k+1)=v_{i}(k)+u_{i}(k)
\end{aligned}
$$

where $r_{i}(k), v_{i}(k), u_{i}(k) \in \mathbb{R}^{d}, x_{i}(k)=\left[r_{i}^{T}(k), v_{i}^{T}(k)\right]^{T}$ is the state of the $i$-th agent and $x_{i}(0)=x_{i 0}$ are the initial conditions. We also define the collective vectors $r(k)=\left[r_{1}^{T}(k), \cdots, r_{n}^{T}(k)\right]^{T}, v(k)=\left[v_{1}^{T}(k), \cdots, v_{n}^{T}(k)\right]^{T}$ and $x(k)=\left[x_{1}^{T}(k), \cdots, x_{n}^{T}(k)\right]^{T}$. The states $r_{i}$ and $v_{i}$ can be used, for example, to describe position and velocity of a point mass moving in a $d$-dimensional space. Next, we present an extension of the contractive technique proposed in Section IV for guaranteeing consensus. For system (38) we adopt the following definition of consensus that is mildly weaker than Definition 3. 
Definition 4 The multi-agent system (38) asymptotically reaches $\mathrm{r}$-consensus if the associated set of equilibria is given by

$$
\begin{aligned}
\Phi_{0}= & \left\{\left[r_{1}^{T}, v_{1}^{T}, \cdots, r_{n}^{T}, v_{n}^{T}\right]^{T}:\right. \\
& \left.r_{1}=r_{2}=\cdots=r_{n}, v_{1}=v_{2}=\cdots=v_{n}=0\right\}
\end{aligned}
$$

and for all initial conditions, the states converge to a point in $\Phi_{0}$ as $k \rightarrow+\infty$.

Correspondingly, we define $z_{i}^{r}(k)$ as

$$
z_{i}^{r}(k)=K_{i}(G(k)) r(k)
$$

and introduce the following cost for agent $i$ :

$$
\begin{aligned}
& J_{i}\left(x_{i}(k), z_{i}^{r}(p(k) N), U_{i}(k)\right)= \\
& J_{i}^{r}\left(r_{i}(k), z_{i}^{r}(p(k) N), U_{i}(k)\right)+J_{i}^{v}\left(v(k), U_{i}(k)\right)+J_{i}^{u}\left(U_{i}(k)\right)
\end{aligned}
$$

where

$$
\begin{gathered}
J_{i}^{r}\left(r_{i}(k), z_{i}^{r}(p(k) N) U_{i}(k)\right)= \\
\alpha_{i} \sum_{j=2}^{N+1}\left\|r_{i}(k+j)-z_{i}^{r}(p(k) N)\right\|^{2} \\
J_{i}^{v}\left(v(k), U_{i}(k)\right)=\beta_{i} \sum_{j=1}^{N}\left\|v_{i}(k+j)\right\|^{2} \\
J_{i}^{u}\left(U_{i}(k)\right)=\gamma_{i} \sum_{j=0}^{N-1}\left\|u_{i}(k+j)\right\|^{2}
\end{gathered}
$$

and $\alpha_{i}, \beta_{i}, \gamma_{i}>0$. We consider the optimization problem associated to agent $i$

$$
\min _{U_{i}(k)} J_{i}\left(x_{i}(k), z_{i}^{r}(p(k) N), U_{i}(k)\right)
$$

subject to the following constraints:

(I) the agent dynamics (38);

(II) the input constraint $\left\|u_{i}(k+j)\right\| \leq u_{i, \max }, \quad u_{i, \max }>$ $0, j \in[0, N-1]$;

(III) the contractive state constraints

$$
\begin{aligned}
& r_{i}((p(k)+1) N)=z_{i}^{r}(p(k) N) \\
& v_{i}((p(k)+1) N)=0 .
\end{aligned}
$$
by

We denote the corresponding receding-horizon control law

$$
\begin{aligned}
& u_{i}^{R H}(k)=\kappa_{i}^{R H}\left(k, x_{i}(k), z_{i}^{r}(p(k) N)\right), \\
& \kappa_{i}^{R H}\left(k, x_{i}(k), z_{i}^{r}(p(k) N)\right)=u_{i}^{o}(k \mid k)
\end{aligned}
$$

Due to the double-integrator dynamics of the agents, we require that $N \geq 2$ in order to guarantee that system (38) is reachable. The constraints (46a) and (46b) imply that each agent is periodically forced to arrive to the position given by (46a) with zero velocity. We highlight that, the possibility of fulfilling the constraint (46b) depends on the nature of the agent.
Remark 5 Reasoning like in Remark 3, one has that if problem (45) is feasible at times $l N, l \in \mathbb{N}$ then it is feasible at all times. Moreover, from Bellman's optimality principle one has that for all $l \in \mathbb{N}$ the optimal inputs computed by the solving problem (45) at times $l N+j, j \in[1, N-1]$ are given by (26).

Next we formulate a sufficient condition showing that by choosing $N$ big enough, problem (45) is feasible at all time instants.

Lemma 2 For given initial conditions $x_{i 0}, i \in \mathcal{N}_{G}$ and bounds $u_{i, \max }$, there exist integers $N_{i}$ such that if $N \geq$ $\max _{i \in \mathcal{N}_{G}} N_{i}$, the CFTOC problem (45) with constraints (I), (II), (III) is feasible at all times.

Proof: We first highlight the following properties of $i$-th agent:

(i) at time $k$ let $\hat{N}_{i}$ be an integer verifying

$$
\hat{N}_{i} u_{i, \max } \geq\left\|v_{i}(k)\right\|
$$

Then, the input sequence $u_{i}(j)=-\frac{v_{i}(k)}{\hat{N}_{i}}, j \in[k, k+$ $\left.\hat{N}_{i}-1\right]$ fulfills $\left\|u_{i}(j)\right\| \leq u_{i, \max }$ and steers $v_{i}(k)$ to $v_{i}\left(k+\hat{N}_{i}\right)=0$;

(ii) at time $k$ assume that $v_{i}(k)=0$ and choose a target state $\bar{r}_{i} \in \mathbb{R}^{d}$. Let $\tilde{N}_{i}$ be an integer verifying

$$
\tilde{N}_{i}^{2} u_{i, \max } \geq\left\|\bar{r}_{i}-r_{i}(k)\right\|
$$

Then, the input sequence $u_{i}(j), j \in\left[k, k+2 \tilde{N}_{i}-1\right]$ given by

$$
\begin{aligned}
& u_{i}(m)=\frac{\bar{r}_{i}-r_{i}(k)}{\tilde{N}_{i}^{2}}, \quad m \in\left[k, k+\tilde{N}_{i}-1\right] \\
& u_{i}\left(m+\tilde{N}_{i}\right)=-u_{i}(m)
\end{aligned}
$$

fulfills $\left\|u_{i}(j)\right\| \leq u_{i, \max }$, steers $r_{i}(k)$ to $r_{i}(k+$ $\left.2 \tilde{N}_{i}\right)=\bar{r}_{i}$ and guarantees that $v_{i}\left(k+2 \tilde{N}_{i}\right)=0$.

Next, we prove the feasibility of (45) at all times. In view of Remark 5, without loss of generality we can focus on times $k=l N, l \in \mathbb{N}$. At time $k=0$ consider the input sequence $\left[\begin{array}{lll}\mathcal{V}_{i, 1}^{T} & \mathcal{V}_{i, 2}^{T} & \mathcal{V}_{i, 3}^{T}\end{array}\right]^{T}$ where

- $\mathcal{V}_{i, 1}$ collects $N_{i, 1}$ input samples chosen as in point (i), for $k=0$. In particular, one has $N_{i, 1} u_{i, \max } \geq\left\|v_{i}(0)\right\|$ and $\mathcal{V}_{i, 1}$ steers $x_{i}(0)$ to $x_{i}\left(N_{i, 1}\right)=\left[\begin{array}{cc}r_{i}^{T}\left(N_{i, 1}\right) & 0^{T}\end{array}\right]^{T}$. Note that $N_{i, 1}$ and $r_{i}\left(N_{i, 1}\right)$ depend only upon $x_{i}(0)$ and $u_{i, \max }$.

- $\mathcal{V}_{i, 2}$ collects $N_{i, 2}$ input samples chosen as in point (ii), for $k=N_{i, 1}$ and $\bar{r}_{i}=r_{i}(0)$. Therefore, one has $N_{i, 2}^{2} u_{i, \max } \geq\left\|r_{i}(0)-r_{i}\left(N_{i, 1}\right)\right\|$ and hence $N_{i, 2}$ depends only upon $x_{i}(0)$ and $u_{i, \max }$. Moreover, it holds $v_{i}\left(N_{i, 1}+2 N_{i, 2}\right)=0$.

- $\mathcal{V}_{i, 3}$ collects $N_{i, 3}$ input samples chosen as in point (ii), for $k=N_{i, 1}+2 N_{i, 2}$ and $\bar{r}_{i}=z_{i}^{r}(0)$. In particular, we pick $N_{i, 3}$ verifying

$$
N_{i, 3}^{2} u_{i, \max } \geq d_{\max }=\max _{i, j \in \mathcal{N}_{G}}\left\|r_{i}(0)-r_{j}(0)\right\|
$$


In order to show that this choice is possible, one has to prove that

$$
N_{i, 3}^{2} u_{i, \max } \geq\left\|z_{i}^{r}(0)-r_{i}(0)\right\|
$$

as required by (49).

To this purpose, we highlight that for all $l \in \mathbb{N}$ and $N>0$ one has $z_{i}(l N) \in \mathrm{Co}\left(\left\{r_{i}(l N)\right\} \cup\left\{r_{j}(l N), j \in\right.\right.$ $\left.\left.\mathcal{N}_{i}(G(l N))\right\}\right)$ and then

$$
\left\|z_{i}^{r}(l N)-r_{i}(l N)\right\| \leq \max _{i, j \in \mathcal{N}_{G}}\left\|r_{i}(l N)-r_{j}(l N)\right\|
$$

Moreover, for later use, we also highlight that from (46a) one has $r_{i}(l N) \in \operatorname{Co}\left(\left\{r_{i}((l-1) N)\right\} \cup\left\{r_{j}((l-1) N), j \in\right.\right.$ $\left.\left.\mathcal{N}_{i}(G((l-1) N))\right\}\right), \forall l \geq 1$, and hence

$$
\max _{i, j \in \mathcal{N}_{G}}\left\|r_{i}(l N)-r_{j}(l N)\right\| \leq \max _{i, j \in \mathcal{N}_{G}}\left\|r_{i}(0)-r_{j}(0)\right\|
$$

Inequalities (50), (52) and (53) show that $N_{i, 3}$ verifies (51). From (50) it follows that $N_{i, 3}$ depends only upon $x_{i}(0)$ and $u_{i, \max }$.

Hereafter, we set $N_{i}=N_{i, 1}+2 N_{i, 2}+2 N_{i, 3}, N=$ $\max _{i \in \mathcal{N}_{G}} N_{i}$ and show a way to build feasible input sequences of $N$ samples at times $l N, l \geq 1$. In particular, for the $i$-th agent we consider the input sequence $\left[\begin{array}{cc}\tilde{\mathcal{V}}_{i, 1}^{T} & \tilde{\mathcal{V}}_{i, 2}^{T}\end{array}\right]^{T}$ where

- $\tilde{\mathcal{V}}_{i, 1}$ collects $N-2 N_{i, 3}$ input samples equal to zero. Since $v_{i}(l N)=0$, one obtains $r_{i}\left(l N+N-2 N_{i, 3}\right)=r_{i}(l N)$ and $v_{i}\left(l N+N-N_{i, 3}\right)=0$.

- $\tilde{\mathcal{V}}_{i, 2}$ collects $N_{i, 3}$ input samples chosen as in point (ii), for $k=l N+N-2 N_{i, 3}$ and $\bar{r}_{i}=z_{i}^{r}(l N)$. We only need to show that if $N_{i, 3}$ is chosen as in (50), then, it fulfills $N_{i, 3}^{2} u_{i, \max } \geq\left\|z_{i}^{r}(l N)-r_{i}(l N)\right\|$ as required by (49). This easily follows from (52) and (53).

It is now possible to state the main result on consensus of this section. Differently from Theorems 2 and 3 , it is just a sufficient condition to achieve consensus.

Theorem 4 Let $\mathcal{F}=\{G(l N), l \in \mathbb{N}$ be a collection of directed [resp. bidirectional] graphs and assume that $N$ is such that the CFTOC problem (45) with constraints (I), (II) and (III) is feasible at all times. Then, the closed-loop multiagent system given by (38) and (47) asymptotically reaches r-consensus if Assumption 2 [resp. Assumption 3] holds.

In order to give a formal proof of the Theorem, it is first necessary to clarify the geometric properties of the optimal control and state sequences computed at times $l N, l \in \mathbb{N}$. To this purpose we define

$$
e_{i}(l N)=\frac{z_{i}^{r}(l N)-r_{i}(l N)}{\left\|z_{i}^{r}(l N)-r_{i}(l N)\right\|}, \quad \mathcal{R}_{i}(l N)=\operatorname{Span}\left\{e_{i}(l N)\right\}
$$

and denote with $\mathcal{R}_{i}^{\perp}(l N)$ the orthogonal complement of the subspace $\mathcal{R}_{i}(l N)$.

Lemma 3 For $l \geq 1$ and for all $j \in[0, N-1]$ let $x_{i}^{o}(l N+j \mid l N)$ be the state produced by (38) from the initial conditions $r_{i}(l N)=r_{i}^{o}(l N \mid(l-1) N)=z_{i}^{r}((l-1) N)$, $v_{i}(l N)=v_{i}^{o}(l N \mid(l-1) N)=0$ and the input sequence $U_{i}^{o}(l N \mid l N)$. Then

$$
\begin{aligned}
& u_{i}^{o}(l N+j \mid l N) \in \mathcal{R}_{i}(l N) \\
& v_{i}^{o}(l N+j \mid l N) \in \mathcal{R}_{i}(l N) \\
& r_{i}^{o}(l N+j \mid l N)-z_{i}^{r}(l N) \in \mathcal{R}_{i}(l N)
\end{aligned}
$$

Moreover, let

$$
\begin{aligned}
& v_{i}^{o}(l N+j \mid l N)=m_{i}^{o}(l N+j \mid l N) e_{i}(l N) \\
& r_{i}(l N)-z_{i}^{r}(l N)=\mu_{i}(l N) e_{i}(l N)
\end{aligned}
$$

where $m_{i}^{o}(l N+j \mid l N) \in \mathbb{R}, \mu_{i}(l N) \in \mathbb{R}$ and consider the optimization problem

$$
\min _{M_{i}(l N)} \bar{J}_{i}\left(\zeta_{i}(l N), M_{i}(l N)\right)
$$

$$
\begin{aligned}
& \bar{J}_{i}\left(\mu_{i}(l N), M_{i}(l N)\right)= \\
& \alpha_{i} \sum_{j=2}^{N}\left(\mu_{i}(l N)+\sum_{k=0}^{j-1} m_{i}(l N+k)\right)^{2}+ \\
& \beta_{i} \sum_{j=1}^{N} m_{i}^{2}(l N+j)+ \\
& \gamma_{i} \sum_{j=0}^{N-1}\left(m_{i}(l N+j+1)-m_{i}(l N+j)\right)^{2}
\end{aligned}
$$

with $m_{i}(l N+j) \in \mathbb{R}$ and subject to the constraints

$$
\begin{aligned}
& \left|m_{i}(l N+j+1)-m_{i}(l N+j)\right| \leq u_{i, \max }, \\
& \left.m_{i}(l N)=m_{i}((l+1) N)=0, N-1\right] \\
& \mu_{i}(l N)+\sum_{k=0}^{N-1} m_{i}(l N+k)=0
\end{aligned}
$$

Then $\bar{M}_{i}(l N)$ is the optimal solution to (59) if and only if $M_{i}^{o}(l N \mid l N)=\bar{M}_{i}(l N)$.

Proof: In order to simplify the notation, hereafter, for a given $l \in \mathbb{N}$, we use the shifted time axis $k^{\prime}=k-l N$. Using dynamics (38) with initial conditions $v_{i}(0)=0$, one has

$$
\begin{aligned}
& v_{i}(j)=\sum_{k=0}^{j-1} u_{i}(k) \\
& r_{i}(j)=r_{i}(0)+\sum_{k=0}^{j-1} v_{i}(k) \\
& u_{i}(j)=v_{i}(j+1)-v_{i}(j)
\end{aligned}
$$


From (62) one has that (45) is equivalent to the following optimization problem involving only velocity variables

$$
\begin{aligned}
& \min _{V_{i}(0)} L_{i}\left(V_{i}(0), r_{i}(0)-z_{i}(0)\right) \\
& L_{i}\left(V_{i}(0), r_{i}(0)-z_{i}^{r}(0)\right)= \\
& L_{i}^{r}\left(V_{i}(0), r_{i}(0)-z_{i}^{r}(0)\right)+L_{i}^{v}\left(V_{i}(0)\right)+L_{i}^{u}\left(V_{i}(0)\right) \\
& L_{i}^{r}=\alpha_{i} \sum_{j=2}^{N}\left\|r_{i}(0)-z_{i}^{r}(0)+\sum_{k=0}^{j-1} v_{i}(k)\right\|^{2} \\
& L_{i}^{v}=\beta_{i} \sum_{j=1}^{N}\left\|v_{i}(j)\right\|^{2} \\
& L_{i}^{u}=\gamma_{i} \sum_{j=0}^{N-1}\left\|v_{i}(j+1)-v_{i}(j)\right\|^{2}
\end{aligned}
$$

with the constraints

$$
\begin{aligned}
& \left\|v_{i}(j+1)-v_{i}(j)\right\|^{2} \leq u_{i, \max }^{2}, j \in[0, N-1] \\
& v_{i}(N)=0 \\
& r_{i}(0)-z_{i}^{r}(0)+\sum_{k=0}^{N-1} v_{i}(k)=0
\end{aligned}
$$

Using the orthogonal decomposition $v_{i}(j)=\bar{v}_{i}(j)+\tilde{v}_{i}(j)$, $\bar{v}_{i}(j) \in \mathcal{R}_{i}(0), \tilde{v}_{i}(j) \in \mathcal{R}_{i}^{\perp}(0)$ in (63) and (68) we get the optimization problem

$$
\min _{\bar{V}_{i}(0), \tilde{V}_{i}(0)} L_{i}\left(\bar{V}_{i}(0)+\tilde{V}_{i}(0), r_{i}(0)-z_{i}^{r}(0)\right)
$$

with the constraints

$$
\begin{aligned}
& \left\|\bar{v}_{i}(j+1)-\bar{v}_{i}(j)+\tilde{v}_{i}(j+1)-\tilde{v}_{i}(j)\right\|^{2} \leq u_{i, \max }^{2} \\
& \bar{v}_{i}(N)+\tilde{v}_{i}(N)=0 \\
& r_{i}(0)-z_{i}^{r}(0)+\sum_{k=0}^{N-1}\left(\bar{v}_{i}(k)+\tilde{v}_{i}(k)\right)=0
\end{aligned}
$$

Note that if the pair $\left(\bar{V}_{i}(0), \tilde{V}_{i}(0)\right)$ verifies (70), so does $\left(\bar{V}_{i}(0), 0\right)$. Indeed, using the orthogonality of $\bar{v}$ ad $\tilde{v}$;

- constraints (70a) become $\left\|\bar{v}_{i}(j+1)-\bar{v}_{i}(j)\right\|^{2}+$ $\left\|\tilde{v}_{i}(j+1)-\tilde{v}_{i}(j)\right\|^{2} \leq u_{i, \max }^{2}$ and hence are fulfilled by $\left(\bar{V}_{i}(0), 0\right)$;

- constraint (70b) implies $\tilde{v}_{i}(N)=0$, that is verified by $\left(\bar{V}_{i}(0), 0\right)$

- since $r_{i}(0)-z_{i}^{r}(0) \in \mathcal{R}_{i}(0)$, constraint (70c) implies that $\sum_{k=0}^{N-1} \tilde{v}_{i}(k)=0$ that is verified by $\left(\bar{V}_{i}(0), 0\right)$.

From the orthogonality of $\bar{v}$ ad $\tilde{v}$ we also have

$$
\begin{aligned}
& L_{i}\left(\bar{V}_{i}(0)+\tilde{V}_{i}(0), r_{i}(0)-z_{i}^{r}(0)\right) \\
&= L_{i}\left(\bar{V}_{i}(0), r_{i}(0)-z_{i}^{r}(0)\right)+L_{i}^{r}\left(\tilde{V}_{i}(0), 0\right)+ \\
& L_{i}^{v}\left(\tilde{V}_{i}(0)\right)+L_{i}^{u}\left(\tilde{V}_{i}(0)\right) \\
& \geq L_{i}\left(\bar{V}_{i}(0), r_{i}(0)-z_{i}^{r}(0)\right)
\end{aligned}
$$

Hence, the optimal solution $\left(\bar{V}_{i}^{o}(0), \tilde{V}_{i}^{o}(0)\right)$ to (69) verifies $\tilde{V}_{i}^{o}(0)=0$. This proves (55). From (62c) and (62b) also (54) and (56) follow, respectively. In order to complete the proof it is enough to realize that substituting $\bar{v}(j)=m_{i}(j) e_{i}(0)$ and $r_{i}(0)-z_{i}^{r}(0)=\mu_{i}(0) e_{i}(0)$ in $L_{i}\left(\bar{V}_{i}(0), r_{i}(0)-z_{i}^{r}(0)\right)$ one gets the cost $\bar{J}_{i}$ defined in (60). Moreover, substituting the same expressions and $\tilde{v}_{i}(j)=0$ in (70) one obtains the constraints (61).

It is now possible to state the proof of Theorem 4. Note that, differently from the case of single integrators, (56) does not guarantee that $r_{i}^{o}(l N+j) \in \overline{z_{i}^{r}(l N) r_{i}^{o}(l N)}, j \in[0, N-1]$, and therefore for proving Theorem 4 we will use an argument that is substantially different from the one adopted in the proof of Theorem 3.

Proof of Theorem 4: : At times $l N, l \geq 1$, because of the contractive constraints (46) we have

$$
x_{i}((l+1) N)=\left[\begin{array}{cc}
K_{i}(G(l N)) & 0 \\
0 & 0
\end{array}\right] x_{i}(l N)
$$

Since $v_{i}(l N)=0, \forall l \geq 1$, the set of equilibria for (72) is $\Phi_{0}$, defined in (39). It is easy to verify that system (72) verifies Assumption 1. Therefore, the update map (72) is uniformly globally attractive [resp. globally attractive] with respect to $\Phi_{o}$ if and only if Assumption 2 [resp. Assumption 3] holds.

Global attractivity of (72) implies that for all initial conditions $x_{i}(0)=\left[\begin{array}{cc}r_{i}^{T}(0) & 0^{T}\end{array}\right]^{T}$ there exists $\phi \in \Phi_{0}$ such that $x(l N) \rightarrow \phi$ as $l \rightarrow+\infty$. In particular, there exists $\rho \in \mathbb{R}^{d}$ such that $r_{i}(l N) \rightarrow \rho$. From (40) and (2) one also has $z_{i}(l N) \rightarrow \rho$ and hence $r_{i}(l N)-z_{i}^{r}(l N) \rightarrow 0$. By using (58) it follows that $\mu_{i}(l N) \rightarrow 0$.

In view of Lemma 3, for studying convergence of the optimal velocities we consider the optimization problem (59). Note that, for $\mu_{i}(l N)=0$, the optimal solution to (59) is $M_{i}^{o}(l N)=0$. Moreover, since the constraint (61a) can be replaced by the linear constraints $m_{i}(l N+j+1)-m_{i}(l N+$ $j) \leq u_{i, \max }$ and $-m_{i}(l N+j+1)+m_{i}(l N+j) \leq u_{i, \max }$, problem (59) is a multi-parametric quadratic program [24] with parameter $\mu_{i}(l N)$. As shown in [24, Chapter 1.1.4], the optimizer $M_{i}^{0}\left(l N, \mu_{i}(l N)\right)$ is a continuous function of $\mu_{i}(l N)$ and hence $\mu_{i}(l N) \rightarrow 0$ implies that $M_{i}^{0}\left(l N, \mu_{i}(l N)\right) \rightarrow 0$ as $l \rightarrow+\infty$. From (57) and (38b) one has $u_{i}^{o}(l N+$ $j \mid l N)=\left(m_{i}^{0}(l N+j+1 \mid l N)-m_{i}^{0}(l N+j \mid l N)\right) e_{i}(l N)$, $\forall j \in[0, N-1]$ and hence $U_{i}^{o}(l N \mid l N)$ converges to zero.

Let $x^{o}(k)$ be the state given by (38) and (11). From Remark 5 we deduce that $\kappa^{R H}\left(l N+j, x^{o}(l N+j)\right) \rightarrow 0, \forall j \in[0, N-$ 1] and hence, recalling that $v_{i}^{o}(l N)=0$, from (38b) one has that $v_{i}^{o}(l N+j) \rightarrow 0, \forall j \in[0, N-1]$. This also implies, from the equality (38a), $r_{i}^{o}(l N)=z_{i}^{r}((l-1) N)$ and the limit $z_{i}^{r}(l N) \rightarrow \rho$ for $l \rightarrow+\infty$, that also $r_{i}^{o}(l N) \rightarrow \rho$ as $l \rightarrow+\infty$. This concludes the proof.

Reasoning like in Remark 4, it is easy to show that if there exists $l \in \mathbb{N}$ such that $G(l N)$ is complete, $r$-consensus is reached at time $(l+1) N$. Furthermore, also in this case the consensus point depends on the sequence $\mathcal{F}$ of communication graphs.

Example 5 The case we consider includes five agents moving in the plane with the following initial conditions: $r_{1}(0)=$ $\left[\begin{array}{ll}-30 & 30\end{array}\right]^{T}, v_{1}(0)=\left[\begin{array}{ll}-5 & 5\end{array}\right]^{T}, r_{2}(0)=\left[\begin{array}{ll}-25 & 35\end{array}\right]^{T}, v_{2}(0)=$ $\left[\begin{array}{ll}5 & 1\end{array}\right]^{T}, r_{3}(0)=[65-75]^{T}, v_{3}(0)=[-6-5]^{T}, r_{4}(0)=[70-$ $68]^{T}, v_{4}(0)=\left[\begin{array}{ll}-1 & 4\end{array}\right]^{T}, r_{5}(0)=\left[\begin{array}{ll}100 & -25\end{array}\right]^{T}, v_{5}(0)=\left[\begin{array}{ll}2 & 8\end{array}\right]^{T}$. 
The prediction horizon is $N=10$. The weights in the cost function (5) are $\alpha_{i}=\beta_{i}=\gamma_{i}=1$ and the input constraints are $u_{i, \max }=200, i=1, \ldots, 5$. We consider a time-invariant collection of unweighted bidirectional graphs $\mathcal{F}$ described by the matrix (18). The simulation results depicted in Fig. 10 and 11 confirm asymptotic r-consensus.
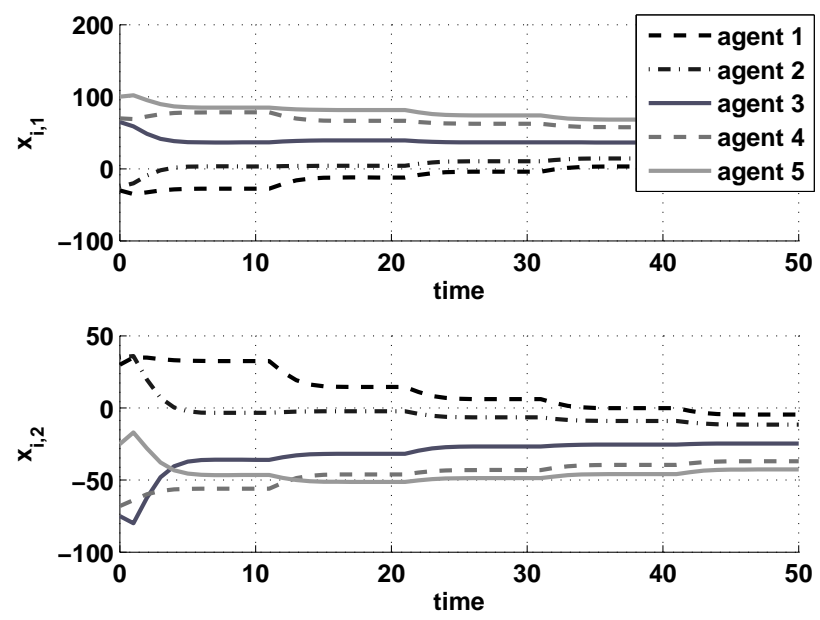

Fig. 10. Example 5: Evolution of agents' position.
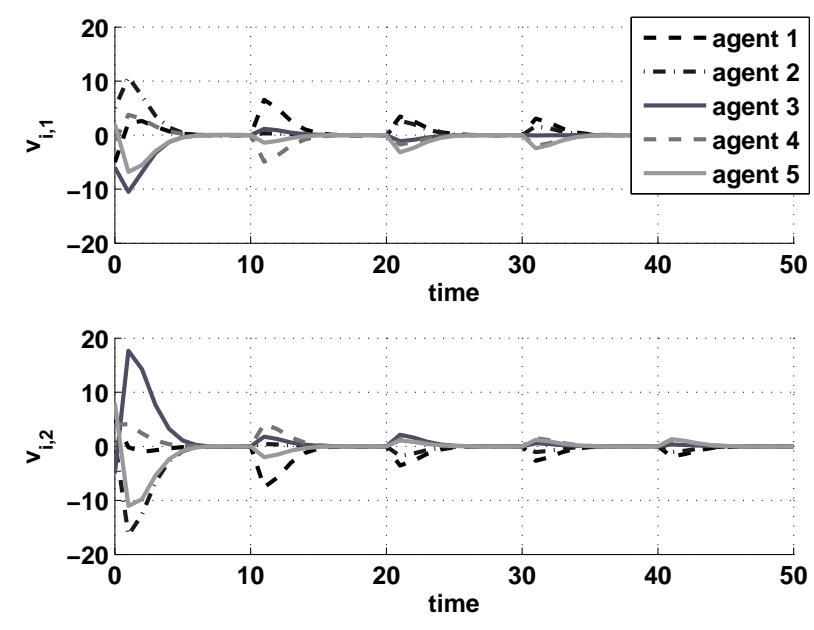

Fig. 11. Example 5: Evolution of agents' velocity.

Remark 6 The control techniques proposed in this paper can be blended to obtain notable group behaviors in the multiagent system. This can be shown by an example. Take $n$ agents with double-integrator dynamics, moving in a threedimensional space. We apply to these agents:

- the contractive single-integrator solution (Section IV) to obtain consensus on the velocity variable in two directions, by applying suitable accelerations;

- the contractive double-integrator solution presented in this section to obtain consensus on the position variable in the remaining direction.
In this way we (asymptotically) obtain a planar movement of the agents with the alignment of their velocities along that plane. Further combinations of the proposed solutions can be studied, thus realizing other actions of interest.

\section{CONCLUSIONS}

We have proposed MPC control schemes capable of guaranteeing consensus in a multi-agent system where individual dynamics are described by a single- and double-integrator models. The proof of consensus, which holds under suitable assumptions on the communication network, relies on convergence results of [3], applicable because of the particular properties of optimal state trajectories. The generalization of the proposed control techniques to groups of agents with more complex dynamics is not straightforward and will be a topic of future research. Future extensions of this work will also concern an assessment of the effect of communication delays and/or uncertainties on the performance of the proposed control schemes.

\section{APPENDIX}

\section{PROPERTIES OF DISCRETE-TIME PATHS}

Given the points $P_{1}, P_{2} \in \mathbb{R}^{d}$, let $\overline{P_{1} P_{2}}$ be the segment joining them and denote with $\left|\overline{P_{1} P_{2}}\right|$ the segment length. The straight line passing through $P_{1}$ and $P_{2}$ will be denoted with $r_{P_{1} P_{2}}$. An $N$-path is an ordered sequence of $N$ points $T=$ $\left\{P_{1}, P_{2}, \ldots, P_{N}\right\} \subset \mathbb{R}^{d}$.

Definition 5 Two $N$-paths $T_{A}=\left\{A_{1}, \ldots, A_{N}\right\} \subset \mathbb{R}^{d}, T_{B}=$ $\left\{B_{1}, \ldots, B_{N}\right\} \in \mathbb{R}^{d}$ are equivalent with respect to a point $O \in \mathbb{R}^{d}$ if $\left|\overline{A_{j} O}\right|=\left|\overline{B_{j} O}\right|, \quad j=1, \ldots, N$ and we write $T_{A} \stackrel{\circ}{\sim} T_{B}$

Note that $\stackrel{\circ}{\sim}$ is an equivalence relation.

Definition 6 An $N$-path $T=\left\{A_{1}, \ldots, A_{N}\right\} \subset \mathbb{R}^{d}$ is straight if $A_{j} \in r_{A_{1} A_{N}}, \forall j \in\{1, \ldots, N\}$.

Note that, given an $N$-path $T_{A}$ and $O \in \mathbb{R}^{d}$, there always exists a straight $N$-path $T_{B}$ verifying $T_{A} \stackrel{\circ}{\sim} T_{B}$. This property is depicted in Fig. 12.

For $P, Q \in \mathbb{R}^{d}$ let $s_{P Q}$ be a the straight half-line starting from $P$ and passing through $Q$.

Lemma 4 For a given $O \in \mathbb{R}^{d}$, let $T_{A}=\left\{A_{1}, \ldots, A_{N}\right\} \subset$ $\mathbb{R}^{d}$ and $T_{C}=\left\{C_{1}, \ldots, C_{N}\right\} \subset \mathbb{R}^{d}$ be two $N$-paths such that $T_{C} \stackrel{\circ}{\sim} T_{A}$, and $C_{j} \in s_{O C_{1}}, j=1, \ldots, N$. Then $\left|\overline{A_{j} A_{j+1}}\right| \geq$ $\left|\overline{C_{j} C_{j+1}}\right|, j=1, \ldots,(N-1)$.

Proof: Since $C_{j} \in s_{O C_{1}}$ we have two possibilities, for $j=1, \ldots,(N-1)$ :

- if $\left|\overline{C_{j+1} O}\right| \leq\left|\overline{C_{j} O}\right|$ one has

$$
\begin{aligned}
& \left|\overline{A_{j} O}\right| \leq\left|\overline{A_{j+1} O}\right|+\left|\overline{A_{j} A_{j+1}}\right| \\
& \left|\overline{C_{j} O}\right|=\left|\overline{C_{j+1} O}\right|+\left|\overline{C_{j} C_{j+1}}\right|
\end{aligned}
$$




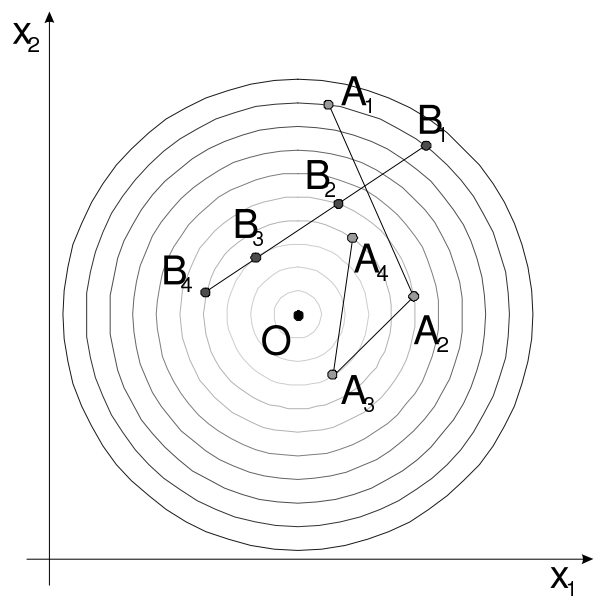

Fig. 12. Two paths $T_{A}$ and $T_{B}$ in $\mathbb{R}^{2}$ equivalent with respect to $O$.

- if $\left|\overline{C_{j+1} O}\right|>\left|\overline{C_{j} O}\right|$ one has

$$
\begin{aligned}
& \left|\overline{A_{j+1} O}\right| \leq\left|\overline{A_{j} O}\right|+\left|\overline{A_{j} A_{j+1}}\right| \\
& \left|\overline{C_{j+1} O}\right|=\left|\overline{C_{j} O}\right|+\left|\overline{C_{j} C_{j+1}}\right|
\end{aligned}
$$

and the result follows immediately from the definition of equivalent trajectories.

We highlight that under the assumptions of the previous Lemma, one has

$$
\sum_{j=1}^{N-1}\left|\overline{A_{j} A_{j+1}}\right|^{2} \geq \sum_{j=1}^{N-1}\left|\overline{C_{j} C_{j+1}}\right|^{2}
$$

Moreover, this inequality is strict if $\exists j: A_{j} \notin s_{O A_{1}}$.

Definition 7 An $N$-path is non-increasing with respect to $O \in$ $\mathbb{R}^{d}$ if $\left|\overline{A_{j+1} O}\right| \leq\left|\overline{A_{j} O}\right|, \forall j \in\{1, \ldots,(N-1)\}$.

Note that if $T_{A}$ is non-increasing w.r.t. $O$ and $T_{B} \stackrel{\circ}{\sim} T_{A}$ then also $T_{B}$ enjoys the same property.

Definition 8 The $N$-path $T_{A}=\left\{A_{1}, \ldots, A_{N}\right\}$ is pointing towards $O \in \mathbb{R}^{d}$ if it is non-increasing w.r.t. $O$ and $A_{j} \in$ $\overline{A_{1} O}, \forall j \in\{1, \ldots, N\}$.

Note that if $T_{A}$ points towards $O$ one has $\left|\overline{A_{1} A_{N}}\right|=$ $\sum_{j=1}^{N-1}\left|\overline{A_{j} A_{j+1}}\right|$.

Theorem 5 Let $T_{A}=\left\{A_{1}, \ldots, A_{N}\right\} \in \mathbb{R}^{d}$ be an $N$ path. Given $O \in \mathbb{R}^{d}$, there always exists an $N$-path $T_{B}=$ $\left\{B_{1}, \ldots, B_{N}\right\} \in \mathbb{R}^{d}$ with $B_{1}=A_{1}$, pointing towards $O$ and satisfying the following inequalities:

$$
\begin{gathered}
\left|\overline{B_{j} O}\right| \leq\left|\overline{A_{j} O}\right|, \quad j=1, \ldots, N \\
\left|\overline{B_{j} B_{j+1}}\right| \leq\left|\overline{A_{j} A_{j+1}}\right|, \quad j=1, \ldots,(N-1)
\end{gathered}
$$

Proof: From Lemma 4 there exists an $N$-path $T_{C}=$ $\left\{C_{1}, \ldots, C_{N}\right\} \subset \mathbb{R}^{d}$ such that $T_{C} \stackrel{\circ}{\sim} T_{A}, C_{1}=A_{1}$ and $C_{j} \in s_{O C_{1}}, j=1, \ldots, N$. We set $B_{1}=C_{1}=A_{1}$ and build up $T_{B}$ in a recursive fashion.

Assume that (74) and (75) hold for all $j \in\{1, \ldots, \bar{j}\}, \bar{j}<N$.

1) If $B_{\bar{j}}=C_{\bar{j}}$ and $\left|\overline{C_{\bar{j}+1} O}\right| \leq\left|\overline{C_{\bar{j}} O}\right|$ we set $B_{\bar{j}+1}=C_{\bar{j}+1}$ we have $\left|\overline{B_{\bar{j}+1} O}\right|=\left|\overline{C_{\bar{j}+1} O}\right|,\left|\overline{B_{\bar{j}} B_{\bar{j}+1}}\right|=\left|\overline{C_{\bar{j}} C_{\bar{j}+1}}\right|$.
2) If $B_{\bar{j}}=C_{\bar{j}}$ and $\left|\overline{C_{\bar{j}+1} O}\right|>\left|\overline{C_{\bar{j}} O}\right|$, it results $\left|\overline{B_{\bar{j}+1} O}\right|<$ $\left|\overline{C_{\bar{j}+1} O}\right|$ for any choice of $B_{\bar{j}+1}$ such that $\left|\overline{B_{\bar{j}} B_{\bar{j}+1}}\right| \leq$ $\left|\overline{C_{\bar{j}} C_{\bar{j}+1}}\right|,\left|\overline{B_{\bar{j}+1} O}\right| \leq\left|\overline{B_{\bar{j}} O}\right|$ and $B_{\bar{j}+1} \in \overline{A_{1} O}$.

3) If $B_{\bar{j}} \neq C_{\bar{j}}$ and $\left|\overline{C_{\bar{j}+1} O}\right| \leq\left|\overline{B_{\bar{j}} O}\right|$ we set $B_{\bar{j}+1}=$ $C_{\bar{j}+1}$; in this way we have $\left|\overline{B_{\bar{j}+1} O}\right|=\left|\overline{C_{\bar{j}+1} O}\right|$ and $\left|\overline{B_{\bar{j}} B_{\bar{j}+1}}\right|<\left|\overline{C_{\bar{j}} C_{\bar{j}+1}}\right|$. The latter inequality, in particular, follows from the fact that, by construction, necessarily $\left|\overline{C_{\bar{j}} O}\right|>\left|\overline{B_{\bar{j}} O}\right|$.

4) If $B_{\bar{j}} \neq C_{\bar{j}}$ and $\left|\overline{C_{\bar{j}+1} O}\right|>\left|\overline{B_{\bar{j}} O}\right|$, one has $\left|\overline{B_{\bar{j}+1} O}\right|<$ $\left|\overline{C_{\bar{j}+1} O}\right|$ for any choice of $B_{\bar{j}+1}$ satisfying $\left|\overline{B_{\bar{j}} B_{\bar{j}+1}}\right| \leq$ $\left|\overline{C_{\bar{j}} C_{\bar{j}+1}}\right|,\left|\overline{B_{\bar{j}+1} O}\right| \leq\left|\overline{B_{\bar{j}} O}\right|$ and $B_{\bar{j}+1} \in \overline{A_{1} O}$.

For the auxiliary trajectory $T_{C}$, recalling that $T_{C} \stackrel{\circ}{\sim} T_{A}$, one has $\left|\overline{C_{j} O}\right|=\left|\overline{A_{j} O}\right|, j=1, \ldots, N$. Furthermore, from Lemma 4, the inequalities $\left|\overline{C_{j} C_{j+1}}\right| \leq\left|\overline{A_{j} A_{j+1}}\right|, j=1, \ldots,(N-1)$ follow. Thus, in all cases it is possible to construct an $N$-path $T_{B}$ pointing towards $O$ and verifying (74) and (75).

If the $N$-paths $T_{A}$ and $T_{B}$ fulfill the assumptions of Theorem 5 , one has

$$
\sum_{j=1}^{N}\left|\overline{B_{j} O}\right|^{2} \leq \sum_{j=1}^{N}\left|\overline{A_{j} O}\right|^{2}
$$

\section{ACKNOWLEDGMENTS}

The work has been partially done in the framework of the HYCON Network of Excellence, contract number FP6-IST511368, and has also received partial funding from the European Community Seventh Framework Programme FP7/20072013 under grant agreement n. 223854.

\section{REFERENCES}

[1] R. Olfati-Saber, "Flocking for multi-agent dynamic systems: Algorithms and theory," IEEE Trans. on Automatic Control, vol. 51, no. 3, pp. 401420, 2006.

[2] D. Bauso, L. Giarré, and R. Pesenti, "Lazy consensus for networks with unknown but bounded disturbances," in Proceedings of the $46^{\text {th }}$ IEEE Conference on Decision and Control, 2007.

[3] L. Moreau, "Stability of multi-agent systems with time-dependent communication links," IEEE Trans. on Automatic Control, vol. 50, no. 2, pp. 169-182, 2005.

[4] R. Olfati-Saber and R. Murray, "Consensus problems in networks of agents with switching topology and time-delays," IEEE Trans. on Automatic Control, vol. 49, no. 9, pp. 1520-1533, 2004.

[5] H. Tanner, A. Jadbabaie, and G. J. Pappas, "Stable flocking of mobile agents, part I : Fixed topology," in Proceedings of the $42^{\text {nd }}$ IEEE Conference on Decision and Control, 2003, pp. 2010-2015.

[6] — , "Stable flocking of mobile agents, part II : Dynamic topology," in Proceedings of the $42^{\text {nd }}$ IEEE Conference on Decision and Control, 2003, pp. 2016-2021.

[7] J. Cortes, S. Martinez, and F. Bullo, "Robust rendezvous for mobile autonomous agents via proximity graphs in arbitrary dimensions," IEEE Trans. on Automatic Control, vol. 51, no. 8, pp. 1289-1298, 2006.

[8] G. Ferrari-Trecate, A. Buffa, and M. Gati, "Analysis of coordination in multi-agent systems through partial difference equations," IEEE Trans. on Automatic Control, vol. 51, no. 6, pp. 1058-1063, 2006.

[9] D. Bauso, L. Giarré, and R. Pesenti, "Non-linear protocols for optimal distributed consensus in networks of dynamic agents," System and Control Letters, vol. 55, no. 11, pp. 918-928, 2006.

[10] E. Camponogara, D. Jia, B. H. Krogh, and S. Talukdar, "Distributed model predictive control," Control Systems Magazine, IEEE, vol. 22, no. 1, pp. 44-52, Feb 2002.

[11] W. B. Dunbar and R. M. Murray, "Distributed receding horizon control for multi-vehicle formation stabilization," Automatica, vol. 42, no. 4, pp. 549-558, 2006.

[12] L. Magni and R. Scattolini, "Stabilizing decentralized model predictive control of nonlinear systems," Automatica, vol. 42, pp. 1231-1236, 2006. 
[13] T. Keviczki, F. Borrelli, and G. J. Balas, "Decentralized receding horizon control of large scale dynamically decoupled systems," Automatica, vol. 42, pp. 2105-2115, 2006.

[14] D. Raimondo, L. Magni, and R. Scattolini, "Decentralized MPC of nonlinear systems: an input-to-state stability approach," Int. Journal of Robust and Nonlinear Control, vol. 17, pp. 1651-1667, 2007.

[15] A. Richards and J. P. How, "Robust distributed model predictive control," International Journal of Control, vol. 80, no. 9, pp. 1517-1531, 2007.

[16] E. Franco, L. Magni, T. Parisini, M. Polycarpou, and D. Raimondo, "Cooperative constrained control of distributed agents with nonlinear dynamics and delayed information exchange: a stabilizing recedinghorizon approach," IEEE Trans. on Automatic Control, vol. 53, pp. 324 338, 2008.

[17] B. Johansson, A. Speranzon, M. Johansson, and K. H. Johansson, "Distributed model predictive consensus," in $17^{\text {th }}$ International Symposium on Mathematical Theory of Networks and Systems, Jul. 2006.

[18] D. Q. Mayne, J. B. Rawlings, C. V. Rao, and P. O. M. Scokaert, "Constrained model predictive control: Stability and optimality," Automatica, vol. 36, pp. 789-814, 2000.

[19] G. De Nicolao, L. Magni, and R. Scattolini, "Stability and robustness of nonlinear receding-horizon control," in Nonlinear Model Predictive Control, F. Allgöwer and A. Zheng, Eds. Progress in Systems and Control Theory, Birkhauser Verlag, 2000, pp. 3-22.

[20] S. De Oliveira and M. Morari, "Contractive model predictive control for constrained nonlinear systems," IEEE Trans. on Automatic Control, vol. 45, no. 6, pp. 1053-1071, 2000.

[21] A. Jadbabaie, J. Lin, and A. S. Morse, "Coordination of groups of mobile autonomous agents using nearest neighbor rules," IEEE Trans. on Automatic Control, vol. 48, no. 6, pp. 988-1001, 2003.

[22] D. P. Bertsekas, A. Nedic, and A. E. Ozdaglar, Convex Analysis and Optimization. Athena Scientific, 2003.

[23] D. P. Bertsekas, Dynamic Programming and Optimal Control, Two Volume Set. Athena Scientific, 1995.

[24] F. Borrelli, Constrained Optimal Control of Linear and Hybrid Systems, ser. Lecture Notes in Control and Information Sciences. SpringerVerlag, 2003, vol. 290.

\begin{tabular}{|c|}
\hline \\
\\
PLACE \\
PHOTO \\
HERE \\
\end{tabular}

Giancarlo Ferrari-Trecate received the "Laurea" degree in Computer Engineering in 1995 and the Ph.D. degree in Electronic and Computer Engineering in 1999, both from the University of Pavia. In spring 1998 he was visiting researcher at the Neural Computing Research Group, University of Birmingham, UK. In fall 1998 he joined the Automatic Control Laboratory at ETH (Zurich, Switzerland) as a Postdoctoral fellow. In 1999 he won the grant "assegno di ricerca" at the University of Pavia and in 2000 he was appointed Oberassistent at ETH. In 2002, he joined INRIA (Rocquencourt, France) as a Research Fellow. From March to October 2005 he worked at the Politecnico di Milano supported by the grant "rientro dei cervelli". From November 2005 he is associate professor at DIS, University of Pavia. His research interests include distributed and decentralized control, modeling and analysis of biochemical networks, hybrid systems and Bayesian learning.

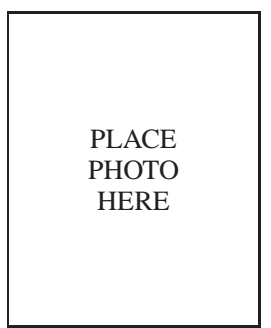

Marco Pietro Enrico Marciandi was born in Milan, Italy, in 1983. In 2006, he received his M.Sc. in Systems and Control Engineering from the Politecnico di Milano with honours. He was awarded the 2006 "Giorgio Quazza" prize by the Politecnico di Milano for his M.Sc. thesis. He is currently at the Italian Research Institute Cesi Ricerca S.p.a., working on the design, realization and test of control systems for test facilities low voltage microgrids, characterised by a strong penetration of distributed energy resources. His current research interests include multiagent systems, decentralized control and the sitting, sizing and economical optimization of renewable energy plants.

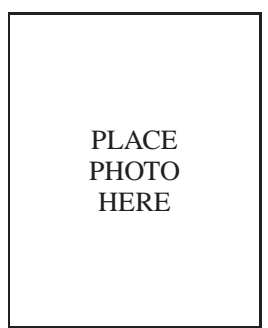

Riccardo Scattolini was born in Milano, Italy, in 1956. In 1979, he received the Laurea degree in Electrical Engineering from the Politecnico di Milano where he is presently professor of Automatic Control. During the academic year 1984/85 he was visiting researcher at the Department of Engineering Science, Oxford University. He also spent one year working in industry on the simulation and control of chemical plants. In 1991 he was awarded the Heaviside Premium of the Institution of Electrical Engineers, United Kingdom. His current research interests include modeling, identification, simulation and control of industrial plants, with emphasis on model predictive control of large-scale systems.
PLACE PHOTO HERE
Luca Galbusera was born in Milano, Italy, in 1982. In 2006, he received the M.Sc. in Systems and Control Engineering with honours from the Politecnico di Milano and was awarded the "Giorgio Quazza" prize for his M.Sc. thesis. He is currently pursuing a Ph.D. degree in Information Engineering at the same institution. His research interests include switched systems, optimal and robust control, model predictive control and multi-agent systems. 